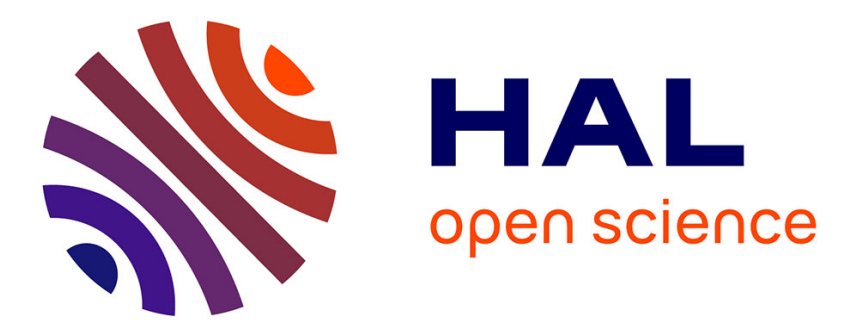

\title{
À propos d'un nouveau monument de Rosmerta
}

Colette Bémont

\section{To cite this version:}

Colette Bémont. À propos d'un nouveau monument de Rosmerta. Gallia - Fouilles et monuments archéologiques en France métropolitaine, 1969, 27 (1), pp.23-44. 10.3406/galia.1969.2517 . hal01934102

\section{HAL Id: hal-01934102 \\ https://hal.science/hal-01934102}

Submitted on 3 Mar 2020

HAL is a multi-disciplinary open access archive for the deposit and dissemination of scientific research documents, whether they are published or not. The documents may come from teaching and research institutions in France or abroad, or from public or private research centers.
L'archive ouverte pluridisciplinaire HAL, est destinée au dépôt et à la diffusion de documents scientifiques de niveau recherche, publiés ou non, émanant des établissements d'enseignement et de recherche français ou étrangers, des laboratoires publics ou privés.

\section{(이) $\$$}

Distributed under a Creative Commons Attribution - NonCommercial - NoDerivatives| 4.0 


\title{
A PROPOS D'UN NOUVEAU MONUMENT DE ROSMERTA
}

\author{
par Colette BÉMONT
}

La découverte récente, à Escolives-Sainte-Camille (Yonne), d'une stèle -- ornée d'un bas-relief surmonté lui-même d'une inscription ${ }^{1}$--- permet d'augmenter un peu la connaissance que nous avons de Rosmerla (fig. 1 et 2a). Cette divinité, parèdre habituelle de Mercure dans le quart nord-est de la Gaule et sur la rive droite de la moyenne vallée du Rhin, où elle est attestée par une série de dédicaces, n'est en effet reconnue, dans sa figuration, pour la quasi totalité des exemples, que par déduction. J'ai tenté, voilà quelques années ${ }^{2}$ de montrer comment on avait pu avec vraisemblance attribuer à la déesse la plupart des monuments (dépourvus d'inscriptions) comportant une figure féminine associée à Mercure ${ }^{3}$, lorsque ceux-ci se trouvaient compris dans l'aire déterminée par les documents épigraphiques (eux-mêmes privés de références iconographiques) ; comment, aussi, dans ces limites, la présence de dédicaces à Maia (autre parèdre de Mercure) rendait douteuse l'identification de certaines représentations, sans qu'aucun critère assuré permît d'établir toujours une distinction; comment, enfin, certaines formes particulières prises par la femme donnée comme compagne à Mercure devaient, au moins d'un point de vue méthodologique, commander quelque prudence, lorsqu'il s'agissait de reconnaître la "grande pourvoyeuse " dans une Vénus, voire une Minerve ou, peut-être, une Hygie ${ }^{4}$. En fait, il existait des présomptions assez solides en faveur de quatre monuments de la région rhénane et de l'Est de la France ${ }^{5}$.

Un cippe d'Ëisenberg' était, jusqu'à la découverte d'Escolives, le seul qui permît, grâce à l'inscription conservée sous le relief, d'identifier sans hésitation une représentation de Rosmerta

1 Cf. R. Kap's, Lne deesse à Essolives-Sainle-Camille, Rosmerla, dans l'Echo d'Auxerre, no 72, novembre-décembre 1967, p. 3-6. L'auteur, qui est le responsable de cette decouverte, donne du relief une description très détaillée. Voici les dimensions de la stèle : haut. $1,52 \mathrm{~m}$; larg. $0,63 / 0,68 \mathrm{~m}$; épais. $0,38 / 0,31 \mathrm{~m}$; taille de la déesse : $1,14 \mathrm{~m}$. Le bloc avait été scié en deux et les morceaux avaient été utilisés dans les fondations d'un etablissement de thermes.

2 Rosmerla, dans Eludes celliques, $I X, \mathrm{n}^{\circ} 17,1960$, p. 29-43.

3 Quand il ne s'agit pas de couples bien connus comme Vercure et Forluna-Tyche.

4 Venus: E. V, 4130 Messancy) ; VI, 5554 'Schweighausen ; Mercure douteux). Minerue: E. X, 7640 'Rimburg; Mercure douteux). Iygie?: E. G., 428 ,Mannheim).

5 Loc. cit., p. 32 et 35 : Met\% (E. V, 4288); Niedaltdorf E. VI, 5lo5, mis lui-même en rapport avec E. V, 4488, à Kirkelneuhäusel); Eisenberg (E. VIII, 6054).

6 Ibid., p. 31-32 et fig. 1, p. 33 = E. VIII, 6039 et $C I L$, XIII, 11696). 
(fig. 2(). Malheureusement cette certitude n'élait que de faible conséquence : le lype d'liisenberg, en effet, demeure peu attesté7, tandis que les moyens propres à sugugérer le pouvoir de la déesse -.-ou son association avec Mercure, dieu de la prospérité sont relativement nombreux (en même lemps, souvent, que peu spécifiques). W. Schleiermacher ${ }^{8}$, d'ailleurs, a montré, en partant de documents de la région du Rhin, que l'iconographie de Rosmerla devait relever de deux types originels principaux : l'un où la divinité aurait comme altributs la patire et la corne d'abondance, l'autre où sa participation à la puissance bienfaisante de Vercure s'exprimerait par la possession de la bourse et du caducée. Il y aurait eu, ensuite, contamination des deux modìles. La pierre d'Wisenberó présente, justement, une de ces figurations composites.

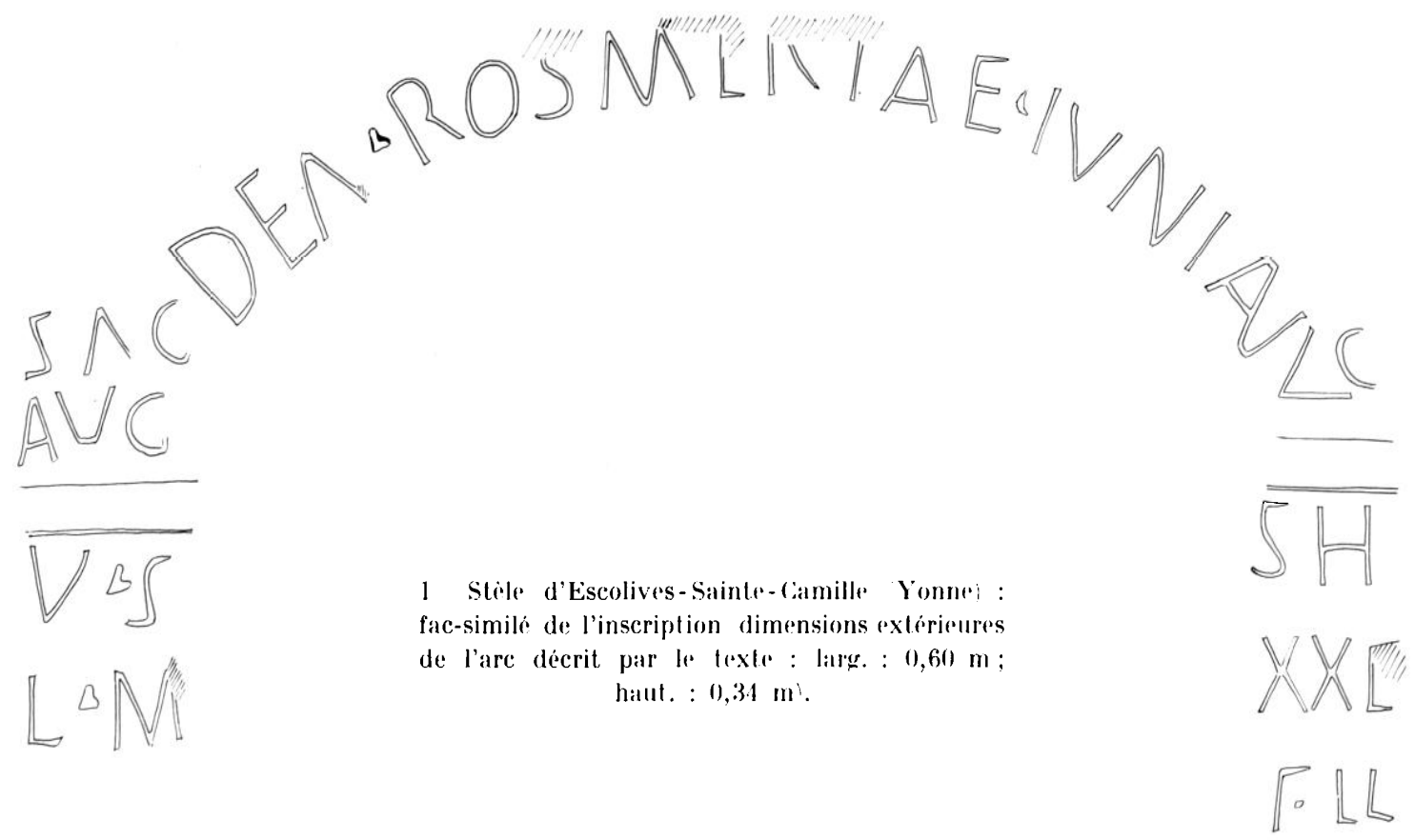

La stèle d'Escolives mérite l'attention à cause de certaines particularités et aussi par les questions qu'elle conduit à poser. La déesse est représentée en pied, de face, dans une niche terminée en cul-de-four et que surmonte un are surbaissé. L'inscription, selon une coutume très répandue en Gaule, dans le cas de monuments votifs ou funéraires, court le long de l'arc et se continue sous les embryons de chapiteaux, qui marquent le raccord de celui-ci avec les piliers latéraux. Elle appelle quelques remarques par sa disposition et son contenu. On peut voir, le long du bandeau (fig. 1) : IEA ROSM ERT TE IVNIN $\breve{V}$; sur le côté gauche, de haut en bas, au-dessus du chapiteau : $S A C / A V G$, sur le pilier : $V \cdot S / L \cdot M$; sur le pilier de droite : $S H / X X L(?) / I . L L$.

7 Il existe un monument du même type à Nöttingen F. G., 350). D’autres groupes, ou des fragments de reliefs, comportent bien une femme tenant une bourse dans la main gauche, mais la main droite mancpue ou est mutilée (E. VI, 4550 : forêt de Hultenhausen ; F. VII, 5505 : Strasbourg). La presence dı cippe E. VIII, 6039 donne plus de vraisemblance à l'hypothese selon laquelle la stele. E. VIII, 6054, également trouvé à Eisenberge malheureusement incomplete, appartiendrait à la mêmo famille iconographique.

8 Sludien an Göllerlypen der römischen Rheinprocinzen, dans 23. Berichl der römisch-yermanischen Kommission, 1933, p. $109-143$. 
Malgré la gaucherie de la mise en place, la lecture doit se faire, pour la première partic, dans l'ordre suivant : $A V G / S A C / D E A \cdot R O S M E R T A E \cdot I V N I A \breve{V}$. En effel, la place de $S A C A V G$ par rapport au chapiteau incite, malgré la disposition sur deux lignes superposées, dans le prolongement d'un montant, à rattacher ces éléments au registre supérieur de l'inscription. On pourrait cependant se référer aux habitudes des graveurs de ce genre de textes pour objecter que, dans la quasi totalité des cas. la lecture se fait de gauche à droite sur l'arc ou le linteau, puis le long de la colonne de gauche et de celle de droite, en observant soit une symétrie de groupe à groupe ${ }^{9}$, soit, le plus souvent, une continuité ligne à ligne ${ }^{10}$ : en conséquence $S A C / A V G$ devrait se placer après $I V N I A C \breve{V}$. Cette objection, à mon avis, serait assez fragile, pour deux raisons : proposée pour mieux rendre compte de la disposition matérielle, elle négligerait, en fait, et la séparation créée par les chapiteaux et l'absence. d'un pendant, à Iroite, des mots inscrits en haut sur la colonne de gauche; d'autre part, le contenu même du texte scrait alors contestable. Car l'abreviation $S A C / A V G$ ne semble pas correspondre à un titre connu qu'on puisse attribuer au dédicant ${ }^{11}$, pas plus

9 C'est ce qui se passe, par exemple, pour une stiele de Sens E. I1, 2817). L'inscription, gravée dans le champ, comprend deux colonnes, decomposées en groupes de deux ou trois lignes; chaque groupe de gauche a pour suite son pendant dans la colonne de droite, puis on revient à gauche, ete. le tỵpe de symétrie, préféró à l'enchaînement immédiat ligne à ligne de gauche à droite puis, de nouveau, à gauche est, d'apres l'ensemble des monuments publies par

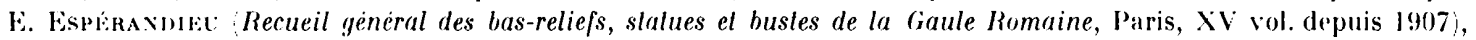
extrêmement rare. On peut signaler, encore, une irrégularité à Reichshoffen : E. VII, 5j9l) : les abriviations $L L, M$, disposées verticalement sur le pilier de droite, font immédiatement suite au texte du linteau.

10 C'est, de loin, la disposition la plus fréquente. Une exception toutefois : la formule $D$ (iis) $M($ anibus) est d'ordinaire extraite de son contexte et ses élements sont placés de part et d'autre du lexte, souvent en dessous cf. E. III, 2312: IV. 3451 ; VI, 4860). Voir, à propos des steles a niche et des inscriptions qu'on y observe, F. Brakurar,

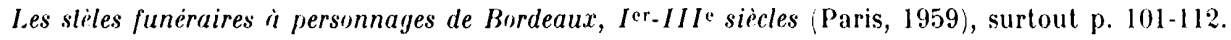

11 Il existe, à ma connaissance, deux exemples, peut-être trois, de sacerdos augusli iou augustalis?), au (CII., XIII, tous dans le voisinage du monument d'Escolives. I.'une des stoles, actuellement à Langres, paraît avoir été trouvée dans la région $(: I I, X I I I, 5688)$; une autre provient des sources de la Seine $\{(1 I I, X I I I, 2870\}$, la dernière du territoire des Ségusiaves Feurs : CIL, XlII, 1642). La premiere, très mutilóe, est restitue comme suit par Th. Nommsen :

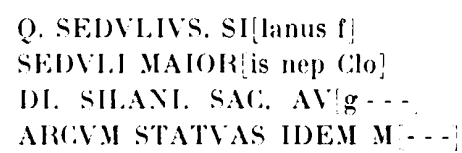

la troisieme liọne peut être complètée en $A V[g u s l i]$ ou $A V$ gustalis $]$ sans quion sache, d'ailleurs, si le texte comportait autre chose qu'une abréviation. La formule $A V G(u s ! i)$ ET ROMAE est à exclure : l'ordre communément attesté est ROMAE ET AVC. De la deuxième dédicace ne subsiste qu'un fragment presque inutilisable :

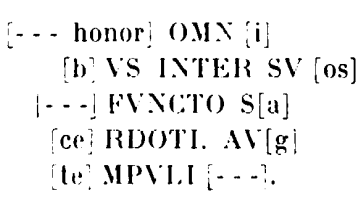

I.a derniere est complete:

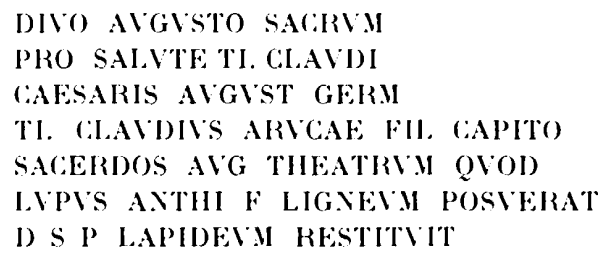

Le seul exemple assuré du titre de sacerdes augusti(?) se présente sous une forme explicite et l'absence d'abréviation de sacerdos est confirmée par l'inscription des sources do la Seine. Toutefois, des libellés plus brefs pouvaient 
que sa place ne se justifie après le nom de celui-ci, si l'on adopte l'interprétation habituelle : $A V G(u s t o) S A C(r u m)^{12}$ (à supposer, d'ailleurs, que l'ordre des deux éléments soit interchangeable $\left.{ }^{13}\right)$. La première ligne se lit donc : $A V G(u s(o) S A C(r u m)$ I)EA(e) ROSMERTAE IVNIT $\widehat{A N} V[s(?) \mid$. Le mot I)EA comporte une faute et doit être rorrigé en DEAE. Le nom du dédicant prête un peu à hésiter : la pente des lettres, en même temps que l'enchaînement du tracé, invite à reconnaître, à la fin du mot, la séquence $A \widehat{N} V$, mais il reste à expliquer la courbe gravée au-dessus de l' $V$. Je n'en ai pas encore trouvé une justification absolument satisfaisante, en admettant qu'on doive bien la considérer comme une lettre. IVNIANVS est possible, car ce qui subsiste du tracé laisse supposer qu'on n'avait pas affaire à un cercle. On pourrait imaginer alors que, faute de place, le lapicide maladroit a tenté ainsi de compléter le nom dont il avait mal calculé les dimensions"'. La colonne de gauche se rétablit sans difficulté : V(olum) S(olvil) L(ibens) $M$ (erilo).

Celle de droite pose quelques problèmes. Voici la suite des lettres : $S H \mid X X L($ ?) $I: L L$. La dernière ligne comporte deux lettres nettes : $L L$, une troisième qui l'est moins : une sorte d'équerre que suit un point. Celui-ci fait-il partie de la lettre? ('est loin d'être assuré, car l'ensemble des caractères de l'inscription ne présente jamais de point à la place des barres. Il s'agit donc plutôt d'une séparation (assez grossière). Il n'est pas impossible de reconnaître, dans le signe $I$, un $F$ et, par analogie avec des formules voisines ${ }^{15}$, $F L L$ peut s'interprèter $F$ (ecil) L(ibens) L(aetus). On peut également y voir un $P$, ce qui, du point de vue du contenu, serait beaucoup plus conforme aux formes traditionnelles (posuil) et, du point de vue graphique, acceptable, à la rigueur. Quelle que soit la solution adoptée, il faut noter la différence entre le texte du pilier de gauche, soigneusement ponctué et gravé et celui du pilier de droite, très imparfait dans sa facture, au point qu'on peut se demander si cette partie de l'inscription n'a pas été faite plus hâtivement, peut-être après coup (ce qui expliquerait, éventuellement la redite maladroite : $L L, V S L M^{16}$.

se rencontrer peut-être plus tardivement), ainsi que le montre le texte de Langres. Mais ce qui rend difficile l'attribution de cette dignité a Ianianus, c'est, d'abord, l'absence des lria nomina -.. alors que les dédicants de Feurs et de Langres sont citoyens romains --- bien que les dimensions du monument d'Escolives expliquent, à la rigueur, une certaine simplification; c'est, surtout, la nature des offandes faites par les deux sacerdoles augusti. L'importance des depenses engagées implique qu'il s'agit de notables, occupant, dans la hierarchie sociale, une situation en vue. Le nom du dédicant d'Escolives, le caractere assez modeste de la stèle excluent, à mon avis, toute comparaison avec ces puissants personnages. Iunianus ne saurait exercer la même charg̣e. Sur la question du sacerdos, de ses rapports avec le famen, de son recrutement dans les provinces, voir, en particulier, C. Ji:l.1A., art. Flamen, dans Dicl. Ant., II 2i, surtout p. $1185-1186$; R. Errwxw, Le culle impérial dans la péninsule ibérique d'Augusle à Dioclétien, Paris, 1958,

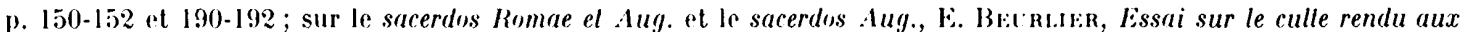
empereurs, Paris, 1890, p. 168-169; sur le sacerdos do Feurs, A. Ar.ml: Rerue épig. du Midi de la lirance II, 1884 1889, fasc. $47, n^{\circ} 699$, p. 349-351:.

12 Lorientation oblique de SAC peut être tenue pour une maladresse mais qui n'exclut pas la volonté de raccorder cette partic de l'inseription à la suite. Les deux mots ont-ils été ajoutés apres coup ? En tout cas, à en juger d'apres la taille des lettres, ces clements du texte ont pu paraitre secondaires à coté d'autres jugés essentiels: le nom de la désse, celui du dédicant.

13 Je n'ai rien trouvé au CIL, XII et XIII qui autorisat cette hypothese.

14 Il n'existe rien de semblable sur l'autre coté du monument et les défauts de la gravure trahissent un manque de methode dans le travail du lapicide, qui semble navoir pas calculé a l'avance l'économie de son texte : apres le I) et l'E, la taille des lettres est réduite, puis, faute de place, $A$ et $N$ sont liés et, bien que l'inscription vienne buter sur le haut du pilier de droite, le nom ne peut être écrit dans son entier.

1) Fecit libens merilo $(: I L$, XIII, 5198). L(ibens) l(aelus) : (:IL, XIII, 6757). L.(ibens) l(aelus) p(osuil) (CIL, XIII, 7356, 8628, 11815). P(osuil) l(ibens) l(aeius) (:II, XIII, 6733, 7275).

16 laut-il imaginer l'influence du modele donné a l'ourrier charge d'ajouter cette mention ? le $P$ se justifierait par son analogic avec l'écriture courante, de même, peut-être, que la disposition des chiffres cf. infra, n. 21 . 
Pour les deux aulres lignes. la présence de chiffres rend raisemblable l'interprétation de SH comme un sigle monétaire. S'agit-il d'une transcription fautive de $I S$, signe correspondant au sesterce? C'est probable : ce ne serait pas le premier exemple de celte erreur ${ }^{17}$ et je n'ai pas trouvé, parmi les interprétations proposées de $S H$, tant par R. Cagnat ${ }^{18}$ que par les Indices du CIL XII et XIII, de solution plus satisfaisante ${ }^{19}$. Les chiffres eux-mèmes font difficulté : les deux $X$ sont parfaitement clairs, mais le signe qui le suit n'est pas net et la lecture est gènée, de surcroît, par une cassure de la pierre ${ }^{20}$. Faut-il reconnaîl re un $L$ ? Les dizaines placées avant les caractères désignant un mulliple de dix sont. d'ordinaire, retranchées de la valeur indiquée par ceux-ci. On se borne, le plus souvent, à signifier par ce moyen la soustraction d'une dizaine, mais il arrive qu'on rencontre, ainsi placés, deux $X$. Les exemples que j'ai relevés ne concernaient qu'un multiple de dix : cent $^{21}$. Cette exclusive s'explique aisément. si l'on conçoit que $X X C$ est une manierre plus rapide de transcrire $L X X X$. Wais on voit mal quel avantage il y aurait a remplacer $X X X$ par $X X L$. Aussi, pour cette inscription, est-on plutòt réduit a deux hypothèses : l'ordre des signes a été modifié, ou la letlre n'est pas un $L$. I.'inversion faulive des caractères se rencontre ${ }^{22}$. On pourrait donc tenir $X X L$ pour l'équivalent de $L X X^{23}$. Cette explication, toutefois, sans ìtre à exclure, ne tient pas compte de l'aspect un peu insolite de cel $L$. En effet, celui-ci parail comporter, en haut de la haste, à droite, un trait horizontal parallèle i la barre inférieure. C'est, du moins, ce qu'on peut déduire de la limitation franche et rectiligne de la cassure de la pierre sur celte arète du pilier. Il est assez peu probable, sinon impossible, qu'on ait un $I$; l'empattement est vraiment très développé vers la droite. Il reste la possibilité de reconnaître en ce signe un $C$ maladroitement tracé24. Cette solution n'est ni absolument satisfaisante, ni assurée, mais l'espacement excessif entre cette lettre et le $X$ et, en conséquence, l'étroitesse du champ libre pour la gravure, peuvent expliquer une gaucherie que l'on retrouve, à des degrés divers, dans le tracé de la courbe supérieure des $S$ (en particulier de celui de la ligne précédente). Quant à la signification, elle ne suscite pas de difficultés, tant pour l'I (XXI=21) que pour le $\Sigma_{-}(X X C=80)$. En fait, seule la connaissance du prix moyen de tels monuments pourrait permettre de choisir à coup sùr, à condition de posséder des termes de comparaison bien établis ${ }^{25}$.

Le contenu de l'inscription mérite quelques observations. La dédicace est faite à Rosmerla seule, associée non à Mercure mais à l'empereur vivant. On ne connait actuellement qu'un autre exemple semblable : un texte gravé sur une petite colonne (aujourd'hui disparue) à Gissey-le-Vieil26, en territoire éduen. L'ofirande est faite alors par Cne. Cominius

17 C.T. CIL, XIII, 86.43.

Is Cours d'épigraphie latine, 4' id., P'aris, 1914, p. 463.

19 On ne rencontre la aucun développement qui puisse correspondre heureusement soit aux modalités de la dédicace, soit aux conditions de l'implantation du monument.

20 L'angle du bloc est écorné et un iclat semble avoir sauté à une époque ancienne.

21 Cf. F. Hervit, Les gra/fites de la Graufesenque, Rodez, 1923, no 8, 1. 8 : X.XC. Le systeme s’applique aussi anx unites, accompagnées ou non d'un signe multiplicatif ibid.: $\left.n^{\circ} 3: 11\right) ; 4,19$; 117 : $\left.I I X\right)$. Ies exemples sont

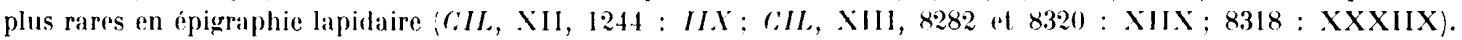

22 CIL, XII, 2085:VIX $=16 ; 5515: I I I X X=23 ;(: I$, XIII, 497:VX = I5.

23 Dans cette iventualite, on ne saurait envisager que difficilement une solution qui justifierait par une seule maladresse l'erreur irlentiqur commise sur les deux lignes successives. Si l'inscription-modele avait été lue à l'envers, la tête en bas, $s, H$ et $X$, lettres symétriques, n'auraient pas cete modifies dans leur trace, seul l'ordre de leur disposition changeait. En cours de travial, la faute dicouverte aurait pu être reparé tant hien que mal. Toutefois, dans cette hypothese, l'ordre des lignes elles-mêmes rit eté modifie : or Sll pricede, comme il est normal, les chiffres.

24 Ce tỵe de lattre se rencontre au CII., XII et XIII. Quand on examine la pixre, une telle lecture paraît tris probable.

25 Or, si l'on a quelques exemples de prix, on ne comnatit pas, en ciaule, de cas où l'on posside à la fois une stiele et la mention dr sa valenr.

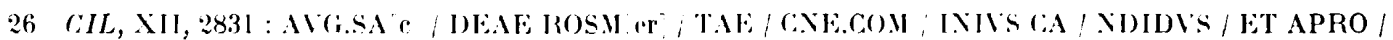
CIA AV/ TIIIA. V.S.I.II. 
Candidus et Apronia Avitilla. On peut noter la distance assez faible qui sépare les deux points où l'existence de tels monuments a été constatée. I)ans un cas, à Gissey, le dédicant est un Gaulois romanisé28, dans l'autre, le simple nom IV VIANV $S^{28}$ ne nous renseigne pas sur le statut social de l'homme. Le loyalisme qu implique le respect du culte impérial s'explique-t-il par le milieu, la fonction ? On ne saurait le dire ${ }^{29}$. En tout cas, cette dédicace, en accomplissement d'un rœu, ne nous en apprend pas plus que la plupart des inscriptions déjà connues sur la nature exacte de la protection accordée par Rosmerla ${ }^{30}$.

La représentation de Rosmerla a un double intérêt : elle s’intègre sans difficulté dans l'une des séries distinguées par W. Schleiermacher ${ }^{31}$. D'autre part, les particularités qu'elle offre permettent de s'interroger sur les rapports qu'entretient la déesse avec les autres figures divines représentées, dans la même région, arec les mêmes attributs.

Rosmerla apparaît, à Escolives, vêtue d'une longue tunique à manches, serrée sous la poilrine par une ceinture; un manteau, drapé en tablier sur le ventre, couvre les épaules et une partie du bras gauche et semble pendre, derrière le corps, jusqu'au sol ; la tête. très mutilée, est coiffée d'un diadème au bord dentelé ; la chevelure, gonflée sur les tempes et cachant les oreilles, retombe en arrière du cou; la main droite, le long du corps, tient une patère (détériorée lors du remploi), comme pour en verser le contenu, tandis que la main gauche soutient la pointe de la corne d'abondance, pleine de fruits sphériques (des pommes ?), qui s'appuie dans le creux du bras.

Cette figuration de la déesse renforce, d'une façon générale. l'opinion que toutes les femmes associées à Mercure et pourvues des mèmes attributs ${ }^{32}$ sont bien Rosmerla. Ce point n'est pas négligeable, puisque, jusqu'a maintenant, les identifications trés probables ou assuréss ne concernaient que des types locaux comportant soit une corbeille de fruits ${ }^{33}$ (tenue pour l'équivalent de la corne d'abondance): soit une bourse (?). une bourse et une corne d'abondance, ou une bourse et une patère $^{34}$. Il n'est pas indifférent, non plus, que ce relief s'intègre dans un groupe, peu nombreux.

27 I.egentilice Cominius est, selon toute apparence, d'origine celliyue. (A. A. Horner, Allcellischer Sprachschatz, Leipzig, I, 1896, p. 1074 et III, 1913, p. 1260-1261, qui cite de nombreux exemples aux t. II, III, V, XII, XIII du CIL.

28 Iunianus admet aussi bien une lacine celtique: 'iun- cf. A. HoI,b:k, op. cil., II, p. xx) qu'une itymologre

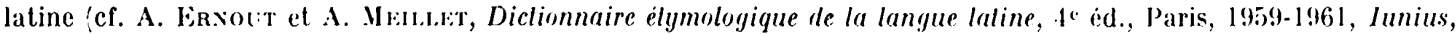
p. $328-329)$.

29 L'inventaire des inscriptions du CIL, XIII, comportant la mention Aug. sar. à coté du nom d'une diviniti indigene montro que les dedicants portent, le plus souvent, les tria nomina mats que l'emploi du nom isole avec parfois la filiation) peut se rencontrer, qual que soit le siecle.

30 Les conditions de decouvertes ne peuvent encore, a l'heure acturlle, donner une qualconque certiturle. Ces blocs utilisés en remploi nont pas dù, apparemment, etant donné leur poids, pareourir une tries longue distance. Il semble normal qu'on les ait reutilises a proximite de l'endroit oì s'elevait lo monument auguel ils appartenaient. L'existence de ce temple était-elle en rapport avec la présence de la source, que l'on roit encore an hordure du territin de fouille? On ne saurait le dire, tant que l'extension du chantier naura pas fourni plus de renseignements sur le nombre et l'implantation des constructions de toutes sortes, qui pouvaient se trouver la. Cf., pour la relation probable

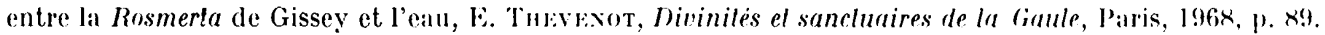

31 Op. cit., p. 113.

32 Rosmerla, p. 40.

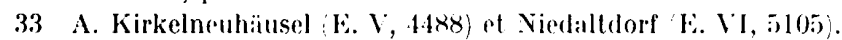

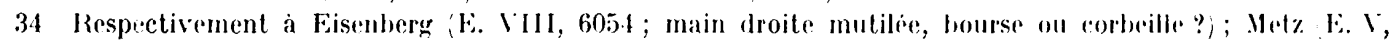
4288); Fisenberg (E. VIJI, 6039). 
certes, mais homogrine. lin effet, on constate que les monuments utilisables de Rosmerla trouvés soit che\% les Éduens, soit che\% leurs proches voisins, ne relèvent que d'un seul type grénéral (fig. 2 a, 3 a el bj: relui d'Escolives ${ }^{35}$. Tandis que dans d'autres régions, la déesse est dotée tantòt des attributs de Nercure tantòt de ceux de Copia. ou encore d'un mélange des uns et des aulres ${ }^{36}$. I a portée de retle remarque ne doit pas être exagérée, car le hasard des fouilles peut être responsable de ce particularisme apparent. d'autant plus que, compte tenu de l'aire intéressée. le nombre des représentations connues est tres réduit. Toutefois, certains traits de la figuration de Maia-Rosmerta révilent. en d'autres lieux, des caractires locaux ou régionaux ${ }^{37}$ et il n'est pas impossible qu'on ait, dans relle zone, préféré un type a un autre ${ }^{38}$. En tout cas, le fait que les monuments puissent être inclus dans des séries contribue a leur faire perdre, plus ou moins, leur raractire fortuit : le couple d'Autun ${ }^{39}$ que la proximité d'Escolives permet, je crois, de comprendre, avec moins de réticence que je ne le faisais auparavant, parmi les reliefs de Mercure et Rosmerla - apparlient au groupe, tris célebre, des couples divins assis, attestés presque exclusivement chez les Éduens el leurs proches voisins ${ }^{40}$. Sil est vrai que l'altitude de la divinité d'Escolives apparente celle-ci a la famille des nombreuses ligures, masculines ou fíminines ${ }^{41}$. portant une corne d'abondance et versant le contenu d'une patere (parfois au-dessus d'un autel), s'il est vrai aussi que ce motif se retroure, pour Rosmerta, à Iandstuhl et a Castel ${ }^{42}$, c'est rependant la déesse de Dijon ${ }^{43}$, sacrifiant au-dessus d'un autel et coiflée d'un diadime, qui. par l'ensemble de ses attributs et de son costume. présente les plus grandes ressemblances aver le relief récemment découvert. On voit ainsi que les monuments de celte région appartiennent soit à un type figuré connu ailleurs mais comportant, dans les cas qui nous occupent, rertaines particularités. soit à des formes iconographiques propres. essentiellement, a la contrée.

Peut-on situer plus précisément la stèle d'Escolives? Les fouilles, d'après les renseignements que m'a aimablement donnés M. R. Kapps, ne permettent pas encore une datation très sûre mais il est permis de penser que le remploi n'est pas intervenu avant le rourant du ir e siècle. Le monument lui-même offre-t-il le moyen de déterminer de façon plus nette les termes chronologiques entre lesquels on peut l'inclure ? Le contenu de l'inscription ne fournit que d'assez minces précisions. Si le long de la vallée du Rhin, de la Moselle et de l'Ahr. l'emploi de la formule In h(onorem) d(omus) d(ivinae) permet de placer

35) II y a trois monuments repartis sur ce territoire : a Escolives, I)ijon E. X, 7519), Autun F. III, I836). Il ne reste malheureusement du relief de Sens, $5.15,2785$ ) qu une description : la deesse portait une corme diabondance de la main gauche, de la droite une "forme de plateau " (une patire ?). Le croissant, qu'on a cru distingurer sur sa tête, pose un problime. Il est trop insolite, à mon avis, pour qu'on puisse, toute verification demenrant impossible, supposer, comme l'a fait W. Schleiermacher ap. cit., p. 114), qu'il s’acrissait d'ailes.

36 A la série dejà commue il faut pent-être ajouter une stèle trouvéc à Soulosse déesse representée, comme celle d'Escolives, debout, avec une patere el une corne dabondance. (f. I. Dénocul, Informations archeologiques, dans Giallia, XVII, 19:9, p. 362, 30.

37 Par exemple, le geste, pour Mereure, de remettre le contenu de la bourse a la déesse fa Coblence et Wiesbaden ; le fait, pour Rosmerla, de sappuyer sur une corne dabondance posee sur le sol chez les Leuques : a Toul et all chatelet, otc.

38 Il nest pas exclu, bien que diflicile a prourer, que le choix s'explique, en fait, par la chronologre.

39 (if. supra, n. 35.

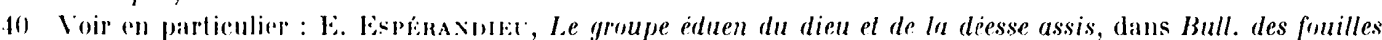
d'Alise, 1, 1914, p. 37.13; J. To(тах, Les diviniles domestiques du pays des Eduens, dans Bull. arch. Comile Trab.

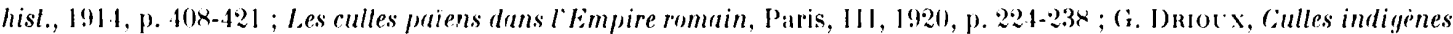

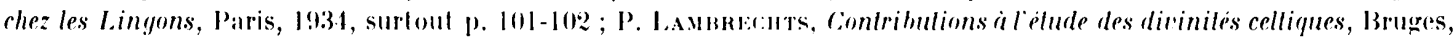

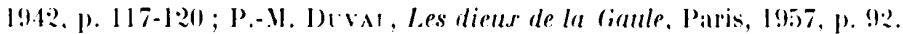

41 Surtout Junon, lo Gienius, les lares.

12 E. VIII, 6069: V11, 5x66.

43 (if. supra п. 35. On ne distingue pas le voile signale par E. Esperandien : en revanche, le motif triangulaire

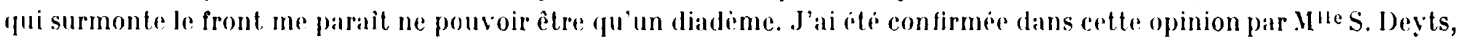
conservatrice du . Wusé arehéologique de Dijon, à lamabilite de qui je dois la photographie de ce monument. 


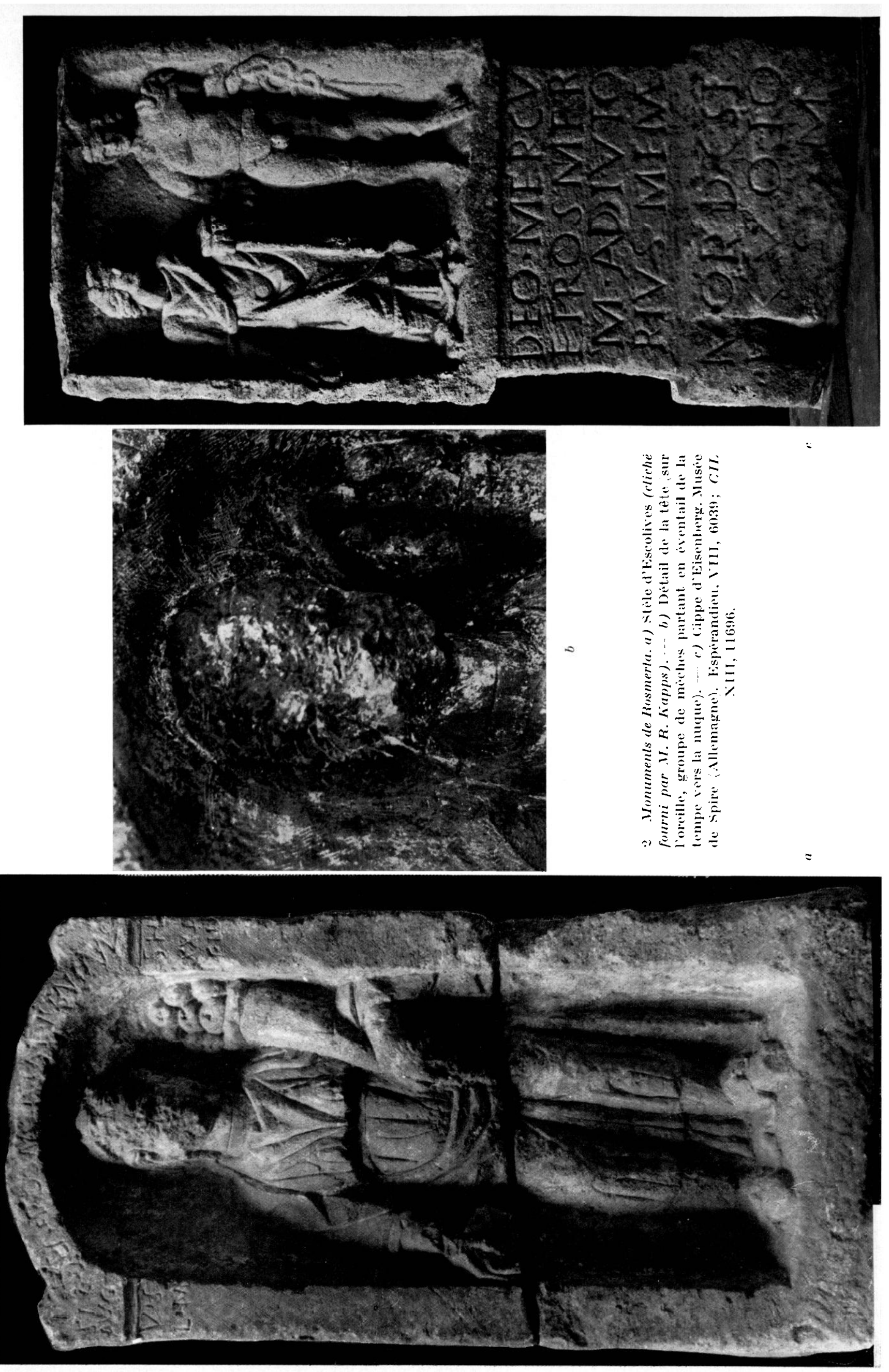


certaines inscriptions ${ }^{44}$ vers 170 ou plus tard ${ }^{45}$, si mème, à Wasserbilligr. une dédicace est latée, par les consuls, de $23: 32^{46}$, ni la formule Aug. sac., ni le redoublement $L$. $L$. n'offrent autant de ressources. L'abréviation $L . L$ semble avoir été utilisée, au moins dans l'expression traditionnelle : VSLLM, concurremment avec la forme plus simple $L$ (dans VSLM) et l'on situe l'apparition de l'une et de l'autre sous Auguste ${ }^{47}$. I) même. le développement du culte impérial commence avec Auguste, la formule Auguslo sacrum s'adresse très vite à l'mupereur vivant, quel qu'il soit ${ }^{48}$, et l'emploi s'en perpétue longtemps ${ }^{49}$.

Lin ce qui roncerne liconographie. W. Sichleiermacher, nous l'avons vu, établit l'existence de deux types: l'un inspiré de la Copia, l'autre de Vlercure lui-mème. Il montre que l'un et l'autre ont élé représentés au moins vers le milieu du $1^{\text {er }}$ siècle ${ }^{50}$. qu'ils ont donné naissance, au cours du

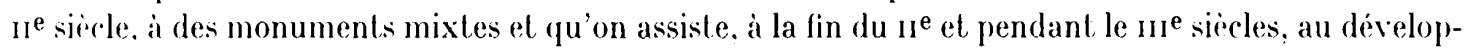
pement des paridres de Mercure présentant l'aspect de déesses-mères ${ }^{51}$ (pour la plupart vètues d'une longue tunique et tenant contre leur poitrine une corbeille de fruils ou une bourse ${ }^{52}$ ). Ce qui n'exclut pas. naturellement, la permanence des modèles préexistants. Ainsi, a s'en tenir à ses attributs, et bien que W. sichleiermacher n'envisage pas très nettement la question de l'apparition de la corne d'abondance el de la patíre. la déesse d'Escolives pourrait se situer entre la seconde moitié du ${ }_{1}{ }^{\text {er }}$ siercle el. au moins, le dernier quart du $\pi^{\mathrm{e}}$ siècle ${ }^{53}$. Toutefois il resterail à montrer que la démonsIration de W. Schleiermacher est toujours valable, dans ses délails, lorsqu'on s'éloigne de la vallée du Rhin, où il choisit ses exemples. De plus. l'absence de llercure nous prive des éléments de datation qu'il pourrait éventuellement donner. Enfin. seuls des termes de comparaison exacts et datés, dans l'iconographie régionale, permettraient de déterminer à quel moment de l'évolution d'un type, ailleurs longuement attesté, on pourrait, situer cette stèle. Car l'imitation de modèles conventionnels empruntés au répertoire romain par des artisans plus ou moins habiles (et cela pendant une durée variable rend malaisée l'appréciation du style.

Compte tenu de ces réserves, la solution la plus sage consiste donc à chercher si des données purement formelles : (aractères de l'inscription, particularités techniques de la sculpture, ne donnent pas, au moins, une idée approximative de la date du monument. Il semble, de l'avis d'un spécialiste ${ }^{54}$, que les sculptures de la stèle ne se distinguent pas par des raracteres eclatants, qui permettent d'en garantir la rate exacte. Ia nature du

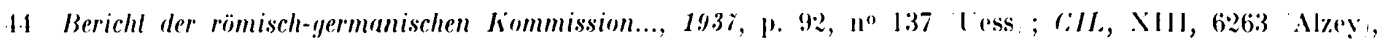

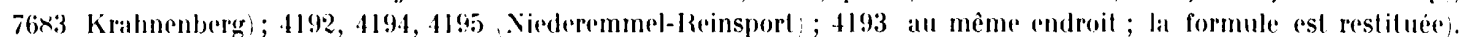

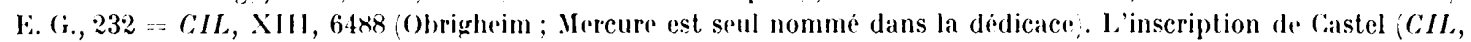
$\mathrm{XIII}, 7270=\mathrm{E}$. VII, 5866; est datée, elle, avec précision, par les consuls, de 170. Gravee sur une pierre à quatre faces dont l'une porte le relief de Mereure et de sa paridres, elle est dédice à Jupiter et Junon.

(5) C'est ce qui ressort, du moins pour les formes abrégées, des articles de Neumann $R E, 1$, art. domus divinu, (.ol. 1527 ot de E. Saglio arl. domus dirina, dans Dict. Anl, II 1), p. 362) faisant suite aux remarques de Th. Iommsen slaatsrecht, 2 e ed., II, p. 792, n. 1 ) at C.. Jullian ! Domus divina, dans Bull. epigr., I1, 1884, p. 251-252)!.

46 CiL, XIII, 4208.

47 R. Civisat, op. cil., p. 253.

tx Sutroxi, Tibere, 26 .

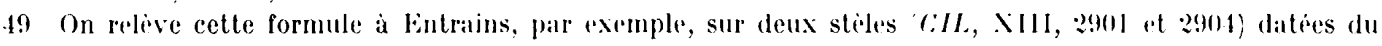
III' siecle.

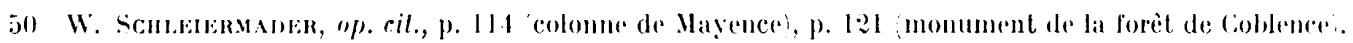

51 Ithid., p. 123-124.

52 Ciest ce qui ressort des exemples cites dans cet article, p. 122.

53 La corme d'abondance est l'attribut de la désse de la forèt de Coblence el l'on retrouve le type à la patere

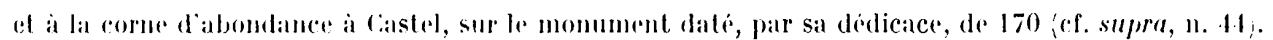

54 J'ai consulte, à er sujet, .1 . F. Brarmer qui ma beaucoup aidée de son experience et de ses conseils. 

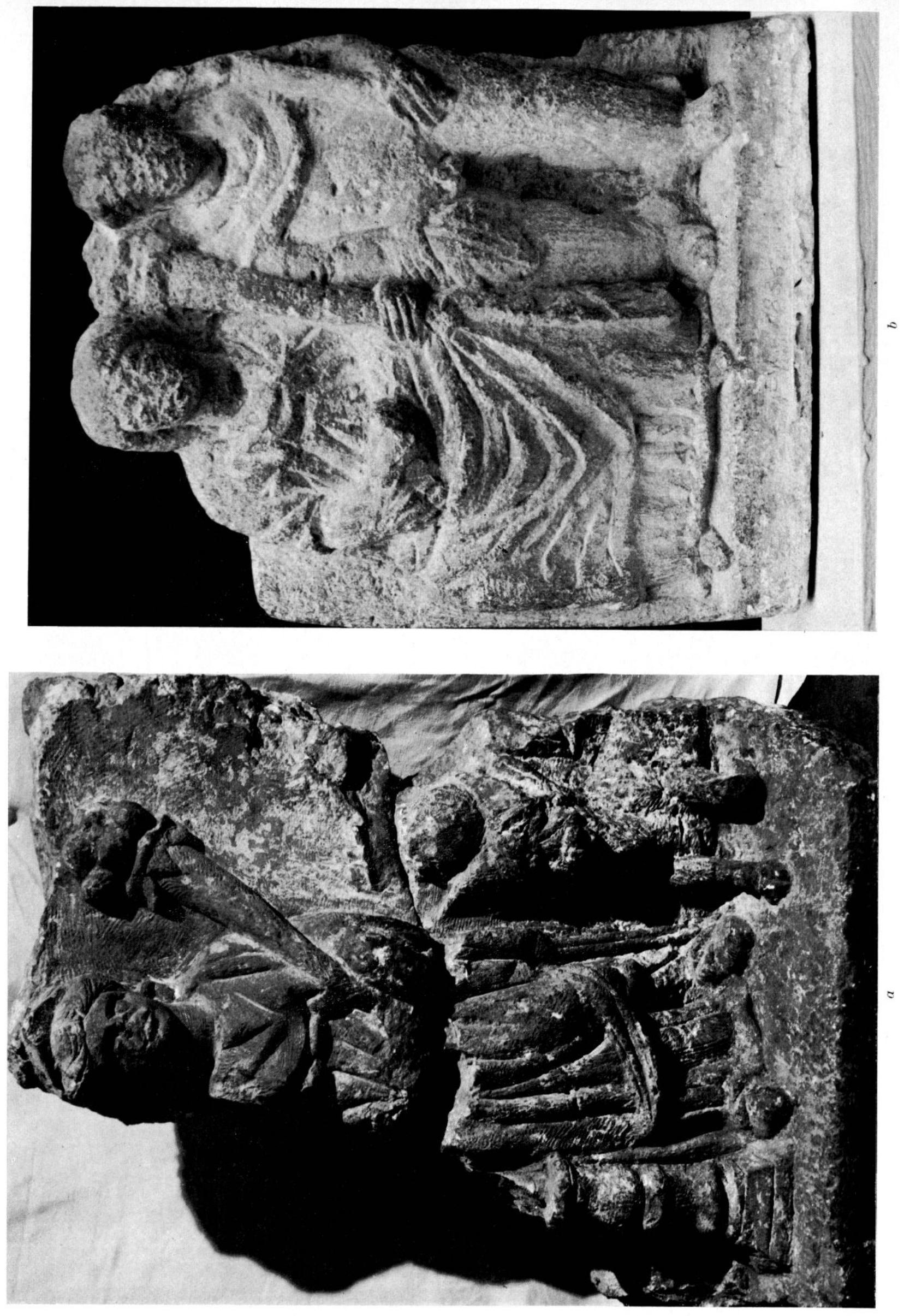
sujet ne prête pas a beauroup de liberté. Néanmoins, l'examen de la stèle ${ }^{55}$ a permis de noter la convergence d'un certain nombre d'indices : forme de la niche, technique des plis, traitement des genoux, qui, tous incitent à placer ce monument entre la moitié du $\mathrm{I}^{\mathrm{e}}$ et la moitié du $\mathrm{u}^{\mathrm{e}}$ siècles et, même, plus précisément, vers 200 . La forme des lettres suppose, elle, une date assez haute56. T'outefois, rien n'autorise à croire que certaines trarlitions graphiques n'ont pu se perpétuer assez longtemps, surtout dans les provinces. In terme chronologique pourrait ètre fixé, cependant, si l'on admet la lecture $\Gamma=P$ : cette forme de lettre est usité au $\mathrm{I}^{\mathrm{er}}$ et $\mathrm{II}^{\mathrm{e}}$ siècles et, apparemment, ne se maintient guire au-delà ${ }^{57}$. I)ans ce cas, le monument se placerait vers la fin du II $^{\mathrm{e}}$ ou le début du III siècle. Il ne s'agit, évidemment, que de probabilités.

line question subsiste, que pose tout particulièrement la découverte de ce monument dans cette région. La dédicace montre que Rosmerla pouvait jouir d'une indépendance plus grande que ne le laissait supposer son habituelle association avec Vercure. Le fait est rendu plus probable par la proximité de l'inscription de Gissey, qui, jusqu'à maintenant, était la seule à proposer une déesse privée de son parèdre. Si le nombre des monuments n'est pas tel que l'on puisse parler d'" habitude", il faut signaler cependant la similitude des formules : Aug. sac. deae Rosmertae et leur caractère apparemment local ${ }^{58}$. L'autonomie de la divinité pourrait toutefois être contestée, grâce aux exemples que l'on possède, ailleurs, de la représentation d'un couple assortie de la dédicace à l'un des dieux ${ }^{59}$. Mais la stìle d'liscolives prouve, puisque Rosmerla est seule figurée, que la déesse a pu, en certains ras, ètre l'objet d'une vénération particulière ${ }^{60}$. La question est donc de savoir ce qu'a ceté, dans cette région, le culte de Rosmerla, ce qu'ont été surtout les limites de l'indépendance de la divinité. Honorée seule, elle peut, théoriquement, être reconnue (à moins d'indications explicitement contraires) dans un certain nombre de représentations féminines isolées portant les mêmes attributs. Dissociée de son parèdre, elle retrouve, en quelque sorte, sa vitalité originelle, et l'on est tenté de s'interroger sur l'identité de toutes les

j) V. Bratemer a releve sur le relief une particularite de la coiffure 'une touffe de petites meches sur l'oreille!, qui paraît propre à ce monument ; cf. fig. 2 b).

56 Je remercie ici $\mathbf{1 1 .}$ J. Mallon, qui a bien voulu examiner l'inscription et m'aider des ressources de son irudition.

57 (:est ce qui ressort de textes paléographiques bien dates ,cf. supra, n. 16), dont le plus récont se situe en 219.

jo Peut-être l'usagre de la formule Augusto sacrum, jointe à la dédicace à une divinité celtique, tient-il à un certain particularisme de cette région : il est intéressant de constater, en effet, que la quasi totalite des exemples de ce genre que j’ai releves au CIL, XIII, proviennent de la même contrée 1575, Le Puy ; 2812, Moulins-Ėngilbert; $2 \times 31$, Gissey-le-Vieil ; 2862-2863, Sources de la Seine ; 2887 , Alise ; $2895,2896,2899,2900$, Sud du territoire des Senons; $2901-2903$, Entrains ; 2921, Auxerre; 11223, 11224, Saint-.1arcel; 11225, 1126, Autun; 11233, Nord du territoire iduen; 11240, 112.41, Mont Auxois . Faut-il y voir le resultat de la rencontre d'un attachement tenace aux cultes indigrines et d'un formalisme romain, imputable au fait que l'on se trouve là dans une zone de passage particulierement active?

59) Il existe trois monuments dediés à Mercure et lo montrant accompagné d'une déesse qui peut être Rosmerta; les dedicaces y sont faites a Mercure seul : Trives : $: I I$, XIII, 7569 et E. VI, 4929; Obrigheim: $1: I L$, XIII, 6488 et

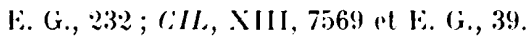

60 Cela ne projuge naturellement pas de son eventuelle association avec un dieu, dans le sanctuaire oì cette stiele a pu ètre dresséc. Mais cette constatation va neamoins a l'encontre de ce que pensait P. I.ambrechts, d'apres les documents dont il disposait alor's "p. cil., Appendice, p. 185; : "Elle n’apparait jamais seule, ni sur les inseriptions, ni sur les reliefs. Elle ne posside aucun attribut qui la distingue spécialement. Elle ne semble pas mener une vie persommelle. Son existence se passe a l'ombre du dieu masculin." 

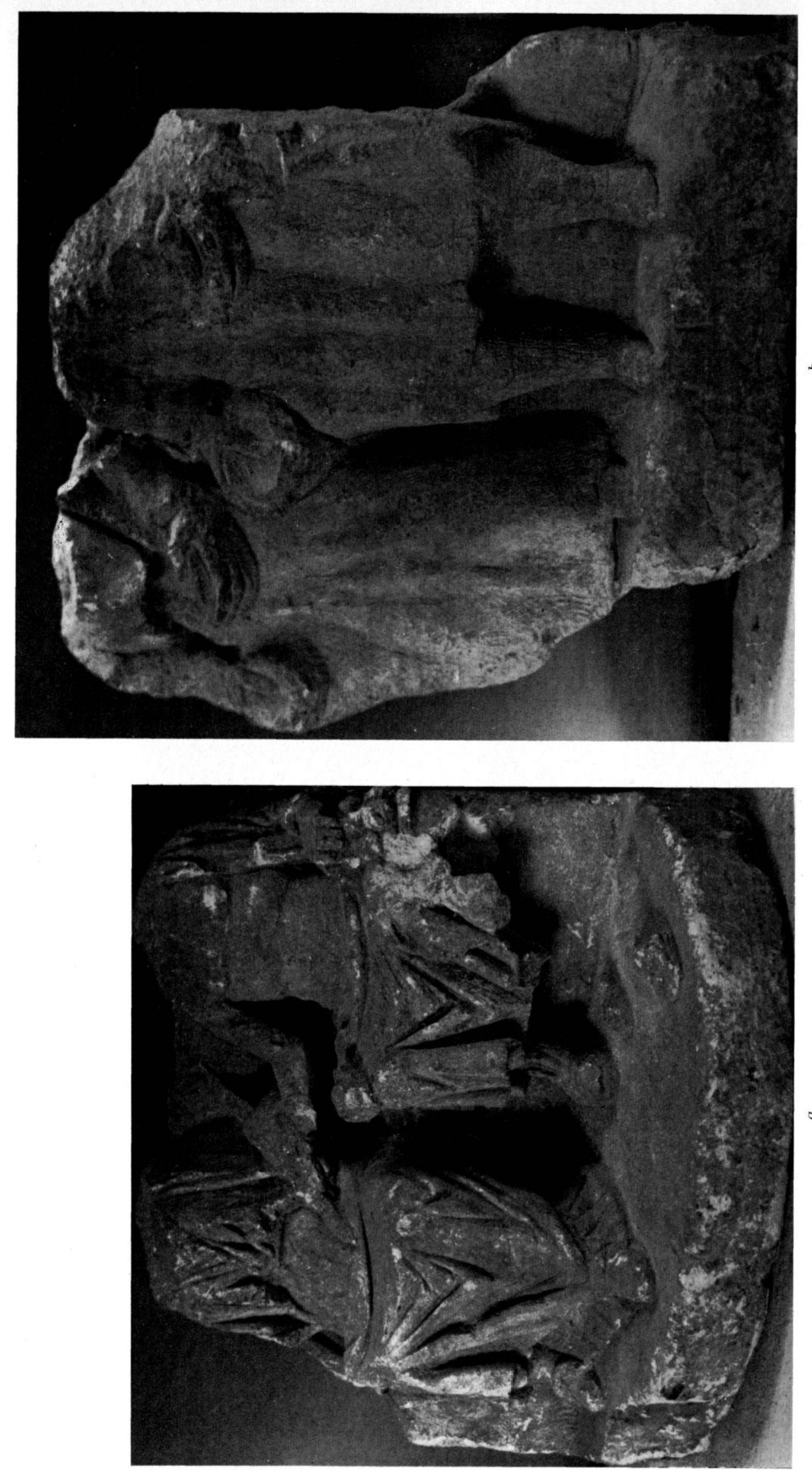

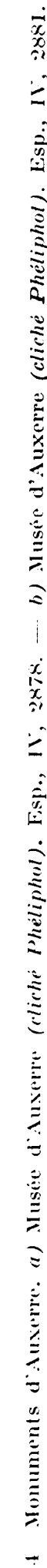


divinités qui, chez les Éduens et leurs voisins, portaient patère et corne d'abondance et se trouraient associées à des figures masculines d'apparences très diverses, en particulier dans le cas des couples assis. Faut-il se fier à la fixité du type de la déesse pour penser que le dieu qui l'ac'compagne représente, sous une forme tantôt romanisée, tantôt indigène, le même personnage, plus ou moins "interprété "? Doit-on, au contraire, s'attacher à la forme très individualisée prise par l'homme et estimer que la représentation féminine I rahit, par l'identité des attributs, l'analogie entre les compagnes de dieux non identifiables les uns aux autres, bien que voisins par leurs prérogatives, en même temps que la pauvreté de limagination des artisans et celle du répertoire iconographique emprunté aux Romains ? Doil-on, enfin, envisager le problème de façon différente selon que le dieu représenté a une forme romaine (particulière ou banale), ou offre des caractéristiques paraissant proprement indigènes? II n'est possible, je crois, que de définir quelques principes de méthorde.

En ce qui concerne les déesses isolées, il ne saurait. à mon avis, ìtre question de retenir des reprisentations autrement différenciées, par exemple par une coillurete. On peut ainsi s'interroger au sujet de la déesse avec patère et corne d'abondance. à Autun. à Chantenay ${ }^{62}$, mais hésiter davanlage a propos des divinités d'Auxerre, saint-Iloré ou Vertault ${ }^{63}$, dont la tête est brisée. Le caractire banal de la patire el de la corne d'abondance attribuées assez souvent (et, en particulier, dans celte région) aux .Matres, à Epon $a^{64}$. permet toutefois difficilement de dire, dans chaque cas, s'il s'agit d'une quelconque Maler topique ou domestique, ou d'une déesse plus largement connue. comme Rosmerta. Seules les conditions de découverte. les inscriptions trouvées à proximité pourraient fournir, au mieux, un argument en faveur de l'une ou l'autre hypothise. En tout cas, on ne saurait aller au-delà des suppositions, en l'absence de dédicaces plus nombreuses à la seule Rosmerta.

Pour les couples, la question intéresse surtout les dieux assis mais pas eux seuls. Il existe, certes, une différence, qu'a bien montrée $J$. Toutain ${ }^{65}$. entre ces modestes reliefs, objets probablement d'un culte domestique, et des stèles ou autels plus importants, dédiés, sans doute, dans des temples et, éventucllement, porteurs d'une dédicace. Ilais il n'y a pas lieu de penser, pour autant, que les divinités concernées dans l'un et l'autre cas étaient nécessairement distinctes. La similitude d'aspect révèle plus que des analogies entre certaines figures assises et d'autres représentées debout, comme le couple trouvé, voilà quelques années, aux Bolards ${ }^{66}$. En ce qui concerne Rosmerta, l'existence d'inscriptions a . Vercure et Rosmerta prouve une association entre les deux divinités chez les Éduens. I)'autre part, la découverte de la stèle d'Escolives montre qu'ì proximité immédiate de cette cité, la déesse pouvait être dotée de la patère el de la corne d'abondance. Ces deux fails incitent à croire que la divinité assise auprès de Mercure, à Lutun, est bien Rosmerta et, corrélativement, que la mème déesse pouvait, chez certains peuples gaulois (comme chez les Romains ${ }^{67}$ ), donner lieu à un culte domestique aussi bien que public. Aussi considérerai-je, dans cette zone, à la fois les petits groupes assis et les stèles de plus grande dimension, assorties ou non d'une inscription ${ }^{68}$.

(i) Ainsi les Tutelles portant la conronne tourelec.

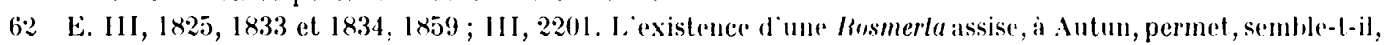
de ne pas accorder une importance décisive a l'attitude de la déesse dans celte riogion.

6.3 F. IV, 2883, 2926, 3375. Les divinites de Bourues 'F. II, 1528) et de Neris (F. 1I, 156.3, avec des fruits dans leur patere, appartiennent à un type un peu différent.

6.1 Matres: par exemple F. III, 1827 Autun); IV, 3373 Vortault: IV, 3593 Hrisey!: Evona: E. III, 21I0

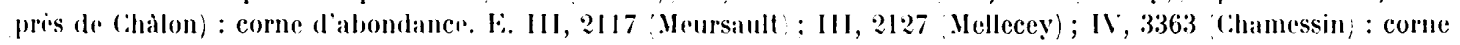
d'abondance et patere.

65) Les diviniles domestiques..., p. 420 .

66 R. Martix, Informalions archénlogiques, dans Gallia, XX11, 1966, p. 386-357 el fig. 19.

67 de que prourent loutes les staturetes on digurines de grandes divinites trouvies, avere les Lares, dans les laraires.

6s la distinction itablie par J. Toutain laisse pendante la question des steles anepigraphes sans contexte

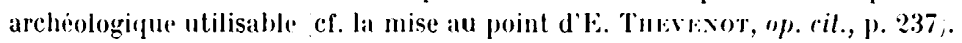



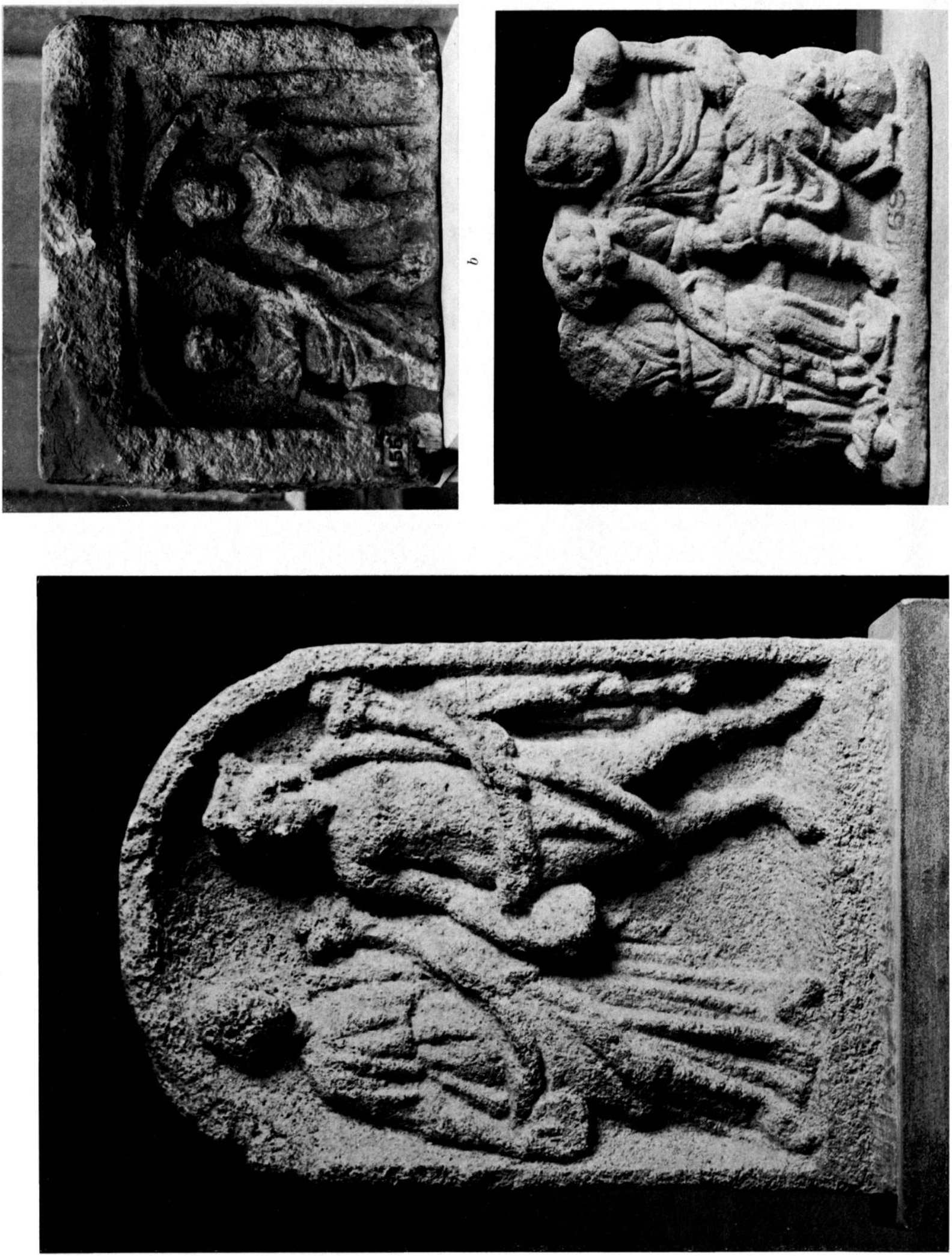
Linventaire permet. dabord, de constater que les groupes assis attestés hors du lerriloire des sénons. des Lingons, des Éduens et des Bituriges se composent d'un dieu, le plus souvent bien dillérencié. el d'une déesse qui ne porte jamais la corne d'abondance et la patere ${ }^{69}$. A l'intérieur de res limites géographiques. en revanche. la déesse tient, dans la grande majorité des cas. ces deux atlributs. C'est leur présence qui m'amene a retenir les couples. assis ou deboul. qui vont nous occuper. Alors, donc, que les femmes ont une apparence semblathle. les divinités masculines se groupent ainsi :

divinites debout : dieu nu identiliable (Genius) (lig. ia ${ }^{70}$.

dieu nu de type militaire (fig. Sh) $)^{71}$;

dieu vìtu identifiable (Sucellus:) ;2 $^{\text {;2 }}$

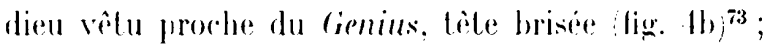

divinités assises : dieu nu identilie Merrure ${ }^{\text {at }}$;

dieu nu de lype militaire

dieu nu avec patire et corne d'abondance. le plus souvent sans tite lign. la ${ }^{76}$; dieu nu non identiliable

dieu vitu fau moins d'une tunique, barbu (quand la tite subsiste, ne portant jamais la patire el la corne d'abondance $e^{78}$ :

dieu vètu doté d'altributs peu reconnaissables ou de caractéristiques insolites ${ }^{79}$.

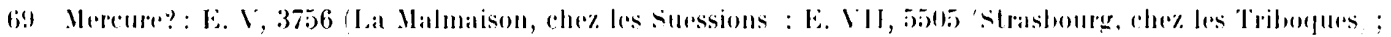

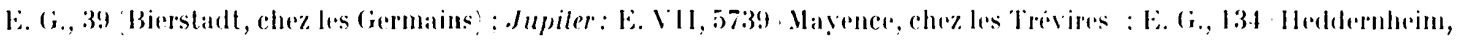
chez les (iermains); Surellus: E. G., 35\% Grïnwinkel, che\% les Germains).

7) E. III, 2023 Chango;.

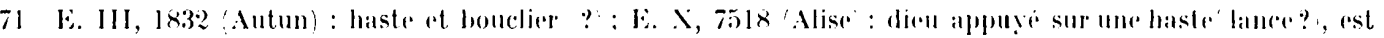
douteux car la deesse ne tient que la corne dahomdance.

72 Aux Bolards, cf. supra, 11. 66.

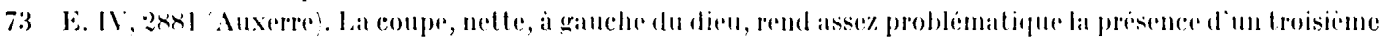
prersollingere.

7.t A Autun, cl. supra, n. 35.

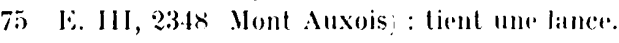

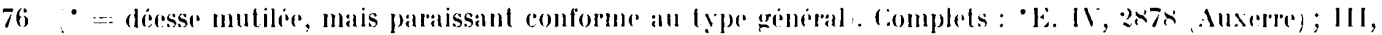

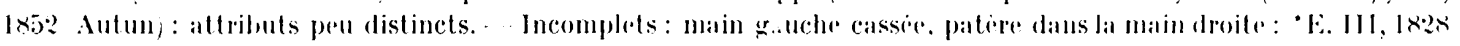

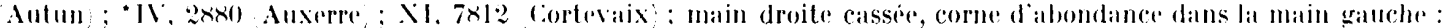
- E. IV, 2911, Ba\%arnes: : IT, 3567 Màlainl.

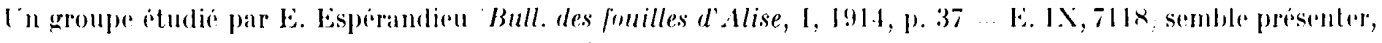

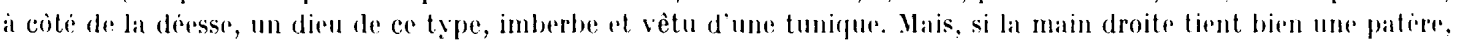
lattribut de gauche a disparu. De plus, des traces de couleur permettent de penser que, sur les crànes lisses et frustes. la chevelure etait peinte : on ne peut done tirer argument du fait que la sculpture ne sugcrere pas l'existence diune barbe. laspect du relief, en général 'bien que la main droite de la figure féminine, tres grossiere, paraisse s'être perdue dans les plis du vêtement, incite plutót a reconnaitre la une mauvaise réplique du monument de 1)ijon E. IT, 34tl : à Dijon, un gros vase est posé sur le sol, entre le dieu barbu au maillet et sa paredre : a Alise, une masse spherigue: occupe, justement, cette place. le même attribut se retrouse encore, au mème endroit, untre les divinitis débout des Bolards cf. n. 66 j. (or, le dieu des Bolards a lapparence du Sucellus traditionnel. I. relief E. III, I K:30 sort des normes: la deesse, amputé de la main droite, pose la gauche sur l'épaule du dieu; de plus un enfint nu était assis a leurs pieds. Jai donc jugé preferable d'écarter ce monument.

77 E. IV, 2879 Auxerre); ・II, la.19 Autun, : les moins mutiles d'une serie de monuments inutilisabhes.

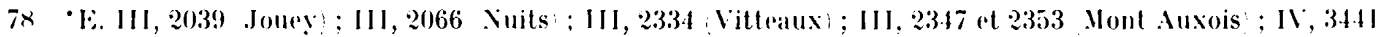

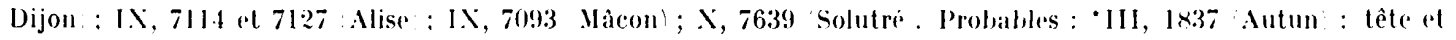
main droite cassées, main gauche appuyee sur une corne dabondance ce. $\mathrm{X}, 7639$, avec une ollet dans la main droile :

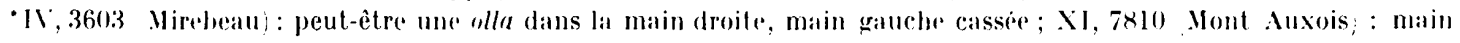
droite cassie, haste couchere sur te hras gauche cf. III, 2039, maillet sur le bras gauche, patere dans la main droite : IX, 7121 Mont Auxois: main gauche casse, patere dans la main droitr II, 1569 Neris : tête cassere, corne dabondance dans la main gauche. main droile?

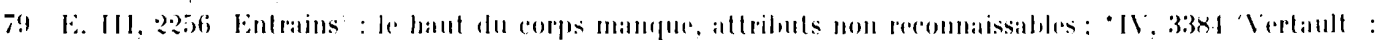

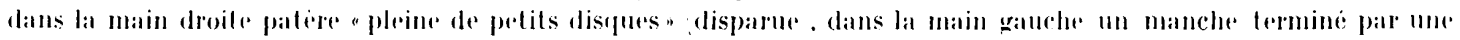

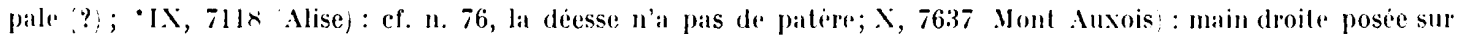
l'epaule de la déesse, petit serpent dans la main gatuche, la déesse n’a pas de pation. 
si l'on envisage la répartition par cité, on conslate un rertain parlicularisme chez les sénons, qui semblent ne connaître que le dieu à la patère et à la corne d'abondance ${ }^{80}$. Ies rares exemples fournis par les Lingons peuvent se partager entre les deux types principaux : le dieu nu avec les attributs de l'abondance. le dieu vêtu et barbu' ${ }^{81}$. Il semble qu'il a pu en ètre de même che\% les Bituriges ${ }^{82}$. Chez les Éduens, enfin, on note le mème mélange aver. en outre, quelques monuments comportant un dieu nu d'allure militaire ou un dieu vêtu difficile à identilier à cause de la nature ou de l'état des attributs qu'il porte etc. Par ailleurs, si la similitude des groupes sénons s'explique peut-itre par la concentralion de ceux-ci entre Auxerre el Bazarnes. il faut remarquer. au contraire. sur les sites éduens où d'abondantes trouvailles ont été faites : le mont Auxois- Alise. Autun, que les représentants des différents types se trouvent mèlés sans qu'il soit possible, étant donné la technique souvent maladroite du sculpteur, de savoir s'il n'intervient pas, entre res monuments divers, un ordre de succession. De plus, on note deux tendances, qui ne font que rendre l'interprétation plus malaisée : on voit -.. je l'ai déjà signalé - -. les mêmes at tributs entre les mains de dieux figurés tantòt debout, tantòt assis; en revanche, l'utilisation des mèmes cartons fait que des dieux, par ailleurs totalement différents, sont représentés avec une telle similitude dans la pose ${ }^{83}$ qu'on est contraint de se demander s'il s'agit d'une mode, ou s'il faut atlacher plus d'importance à la ressemblance dans l'attitude qu'a la différence entre les attributs. Il m'a paru sage, en tout cas. de ne pas accorder a priori une significalion trop grande. dans ces groupes, a l'identilé du type féminin : la corne d'abondance, nous l'avons ru, et la patere ne peuvent ètre considérées comme l'apanage d'une déesse dans cette région.

Il est donc plus juste. à mon avis, de commencer par s'intéresser aux particularités de chaque couple, c'est-i-dire aux attributs de la figure masculine. Celle-ci se ramene, lorsqu'elle n'est pas identifiée avec certitude. a trois formes essentielles : un dieu d'apparence militaire, qui pourrait être un . Mar's local ${ }^{84}$; un dieu vètu et barbu que le maillet. parfois aussi l'olla ${ }^{85}$, rendent très semblable i Sucellus, connu par ailleurs dans le Nord-Est et le Nidi de la Ciaule ${ }^{86}$; un dieu que son rêtement (un manteau atlaché sur l'épaule, laissant le torse largement découvert) el ses attribuls (la patire et la corne d'abondance) apparentent au Genius romain; dans ce cas, toutefois, la détérioration de la lète des monuments conservés ne permet de parler que d'une grande analogie ${ }^{87}$. Les problemes soulevés par les différentes séries se présentent ainsi : il n'est possible, quelquefois, de définir le dieu barbu el vêtu que de façon négative, car il ne porte pas toujours le maillet qui permettrail de l’iden-

si) Compte temu, vo l'etat des monuments, de la similitude physigue, de la presence d'un, an moins, des deux attributs, portes toujours, chacun, dans la même main : E. IN, 2x7x, 2x+(1), $2 \times 81,2911$.

$81 \quad$ \&. IV, 3567 Màlain) : diru nu: 3603 Mircheau: : tres probablement dieu vêu et harbu.

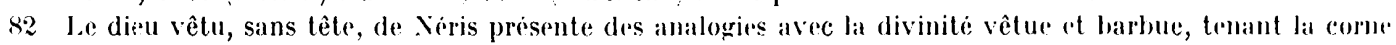
d'abondance et un vase, pue l'on voit a Vitteaux (E. III, 2334: gobelet) et Solutré 'X, 7639): olla). Le personmage figure sur le reliof E. II, 1564 est analogue au dieu nu manteau sur l'epaule, corne d'abondance dans la main gauche. Toutefois l'attribut de la main droite est peu distinct et, surtout, la désse ne tient que la patere, la main gauche étant posé sur l’épaule du dieu. Je n’ai pas cru devoir retenir ce groupe.

83 I.e tabouret sur lequel s’apuie le pied gauche de certains dieux assis n'est lapanage exclusif d'aucune des

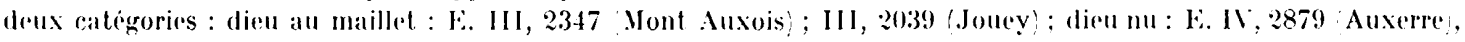
malheureusement mutile.

8.4 Ce pourrait être celui qu'on connaît a Alise sous le nom de Mars Cirol(l)nis. Il est associe tantiot à Bellona Alise et Malain, CIL, XIII, 2472 ot 5598), tantot a Litavis Malain et Aignay-le-1)uc, pries d'Alise, C:IL, XIII, 5601 et 5602,2887$)$.

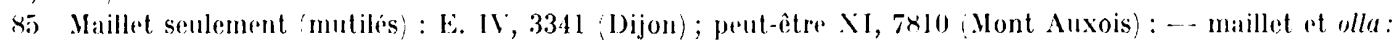
E. IX, 7127 : Alise) ; III, 2066 (Nuits: ; maillet et patere : E. III, 2039 (Joue : ; - maillet et autre attribut : E. III, :2:347 (Mont Auxois : poignard ?) ; — olla seulement (mutile; : peut-être E. IV, 3603 (Wirebeau); - olla et corme d'abondance : E. X, 7639 Solutre ; III, 2334 ? "Vitteaux : un gobelet tronconique remplace l'olla: : - olla at autre attribut: E. III, 2353) (Mont Auxois : bourse); IX, 7114? Alise : tommelet ; l'olla n'est que probable:

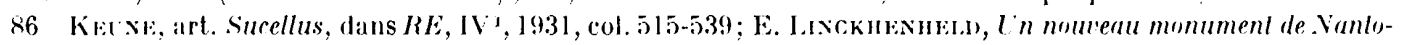

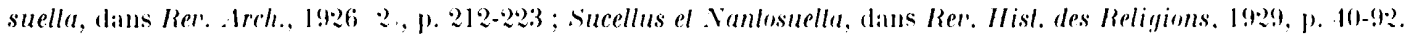

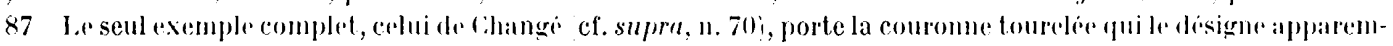
ment comme un protecteur topique. 
lifier, raisonnablement. avec Sucellus. De plus, la présence, parfois. d'un attribul insolile (comme l'épée au Mont Luxois ${ }^{88}$ inspire quelque incertitude sur son identité exacte. Mais tel qu'il est, ce dieu présente une originalité cerlaine, par rapport aux autres divinités, et. l'ensemble de son aspect physique, de son vètement et de ses altribuls le différencie nettement des autres figures masculines beaucoup plus romaines. Il est difficile, en outre, de le considérer, en chaque lieu où il se manifeste, comme un dieu proprement local ou privé, étant donné l'aire de dispersion et la répartition de ses stiles, el la similitude de cert aines d'entre elles ${ }^{89}$. Il s'agit apparemment, d'un point de vue lypologique, de formes dérivées de l'iconographie d'un dieu celtique, ailleurs connu sous le nom de Sucellus ${ }^{90}$. Certains exemplaires sont tout a fait semblables aux représentations du dieu au maillet ${ }^{91}$ et l'on n'est guère porté à hésiter sur leur dénomination. I'autres, en revanche, plus diversifiés. conduisent a se demander si. parfois. l'anecdote ne l'emportait pas et si. en conséquence, les dénominations ou epithètes du dieu n'étaient pas sujeltes a varier. Qui était sa par’̀dre? Ia femme qui, chez les Médiomatriques, accompagne Sucellus est appelée . Vantosuelta ${ }^{92}$. Il n'échappe donc pas que les chances qu'ont les dieux de Bourgogone de se nommer Sucellus conditionnent. tout d'abord, celles qu'ont leurs parèdres de s'appeler lantosuplla. Mais, en outre. les attributs que porte cette déesse, en particulier la hampe surmontée d'une maisonnette ${ }^{93}$, ne ressemblent quire à ceux que nous royons en territoire éduen. Il faut donc supposer soit l'existence d'iconographies locales d'une mème déesse, soit l'association du dieu barbu avec une autre divinité. Celle-ci est, à coup sûr, une protectrice de la prospérité el l'on peut l'imaginer unique ou diverse, autant que l'on congoit comme une ou multiple l'identité de son paridre. Aucun lexte encore ne permet de rien savoir de son nom.

L.e cas des formes nettement romanisées me paraît un peu différent. Il s'agit, dans toutes les occasions, apparemment, de transpositions, d'interprétations romaines d'une réalité gauloise ${ }^{94}$. la superposition des deux divinités n'est pas nécessairement parfaite. el il peut exister, en conséquence, des formes a la fois multiples et approximatives d'un mème dieu indigène. Mercure est l'un des aspects du paridre celtique de Rosmerta; le groupe d'Autun et les inscriptions de Langres et de Magny-Lambert l'attestent dans celle région ${ }^{95}$. On le considere, au mème titre que sa compagne, comme un garant de la prospérilé. Ia question, dans quelques-uns des cas qui nous occupent, est de savoir si nous n'avons pas affaire a un autre mode de représentation d'une mème divinité. Les allinités du Mars gaulois avec des divinités plus pacifiques ont été plusieurs fois soulignées ${ }^{96}$, mais ses représentations, sur nos monuments, comportent des attributs trop dillérents pour que nous puissions établir, du simple point de vue iconographique, le moindre rapport avec Ilercure. En revanche, le dieu semblable au fienius invile à s'interroger: a-t-il, comme ce dernier, les caracteres exclusifs

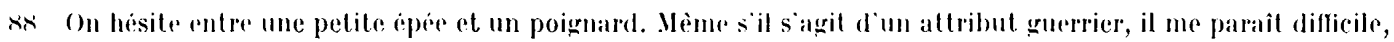
typologifuement, d'envisiger une contamination entre le dieu au maillet el celui qui pourrait être un .Mars local, tant qu'on n'aura pas trouve, a proximite, de groupe où le dieu nu porte une cpese. Autre attribut peu courant bien que, peut-être, moins insolite : le tomneau (E. IX, 7114, Alise); la main droite semble tenir une olla (?; cf. E. III, 2025, cussy-le-Chatel : tonnelet et maillet).

89 Les types produits plus d'une fois (ils ne sont pas tris nombreux) sont rarement groupes ef. infra, n. \$1, 103, 104 ot la répartition d'attributs fréquents, comme le maillet et l'olla, se fait sans ordre apparent.

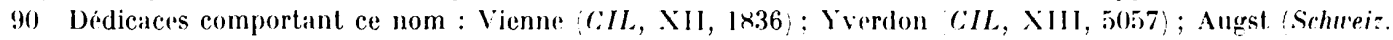
Anz., XXVI, 1924, 205; Worms (CIL, XIII, 6224; Hayence? C:IL, XIII, 6730); Sarrehourg (CII, XIII, 4542; ; Frescaty (Grenier, Revue des Eludes anciennes, XXVI, 1924, p. 136); York Iessau, $46 \times 9$ ).

91 Aux Bolards supra, n. 66); a Nuits (E. III, 2066; ; Alise F. IX. 7127).

92 le que proure la dedicace de Sarrebourge.

93 E. I.s.kexHl:1, sucellus..., p. 59-61.

94 L'association par couples semble caractéristique du nilieu religieux celtipure ce. J. Vavingris, La religion

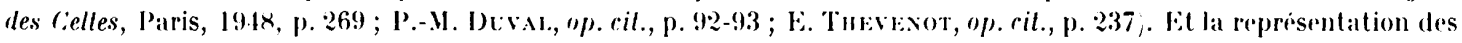
couples divins assis, en particulier, se rencontre surtout, compte tenu des momuments acturellement connus, che\% les Eduens et leurs proches voisins.

$9 \bar{j} \quad$ (if. supra, n. 35, CII, XIJI, 5677 et F. III, 2336.

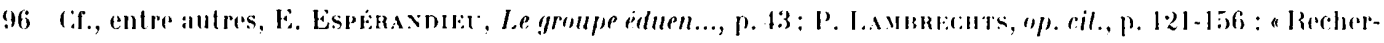
ches sur le culte de llarenre et de llars en Giaules. 


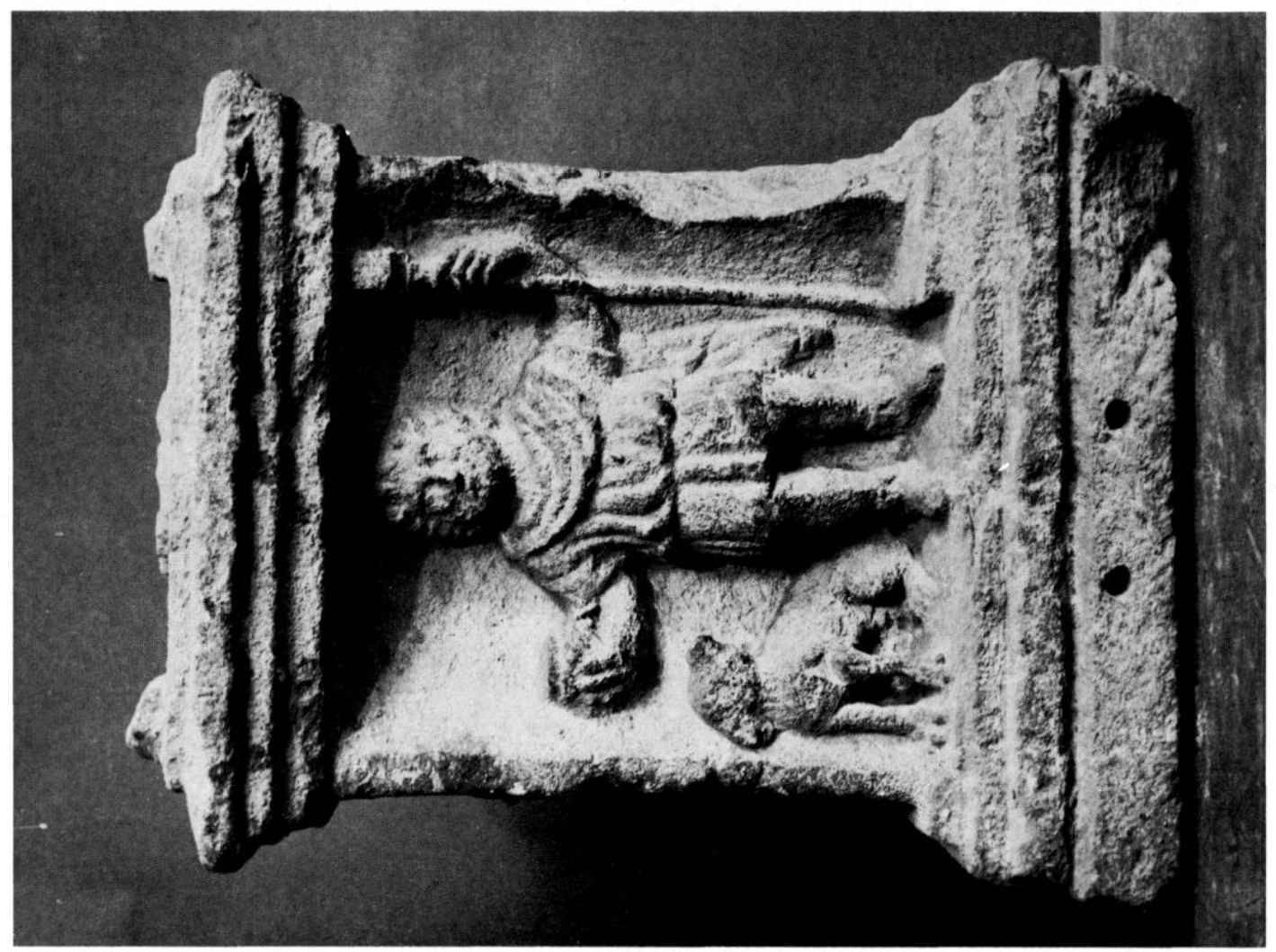

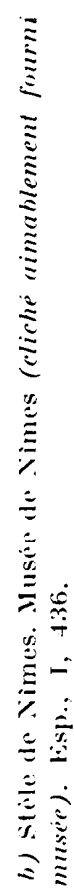

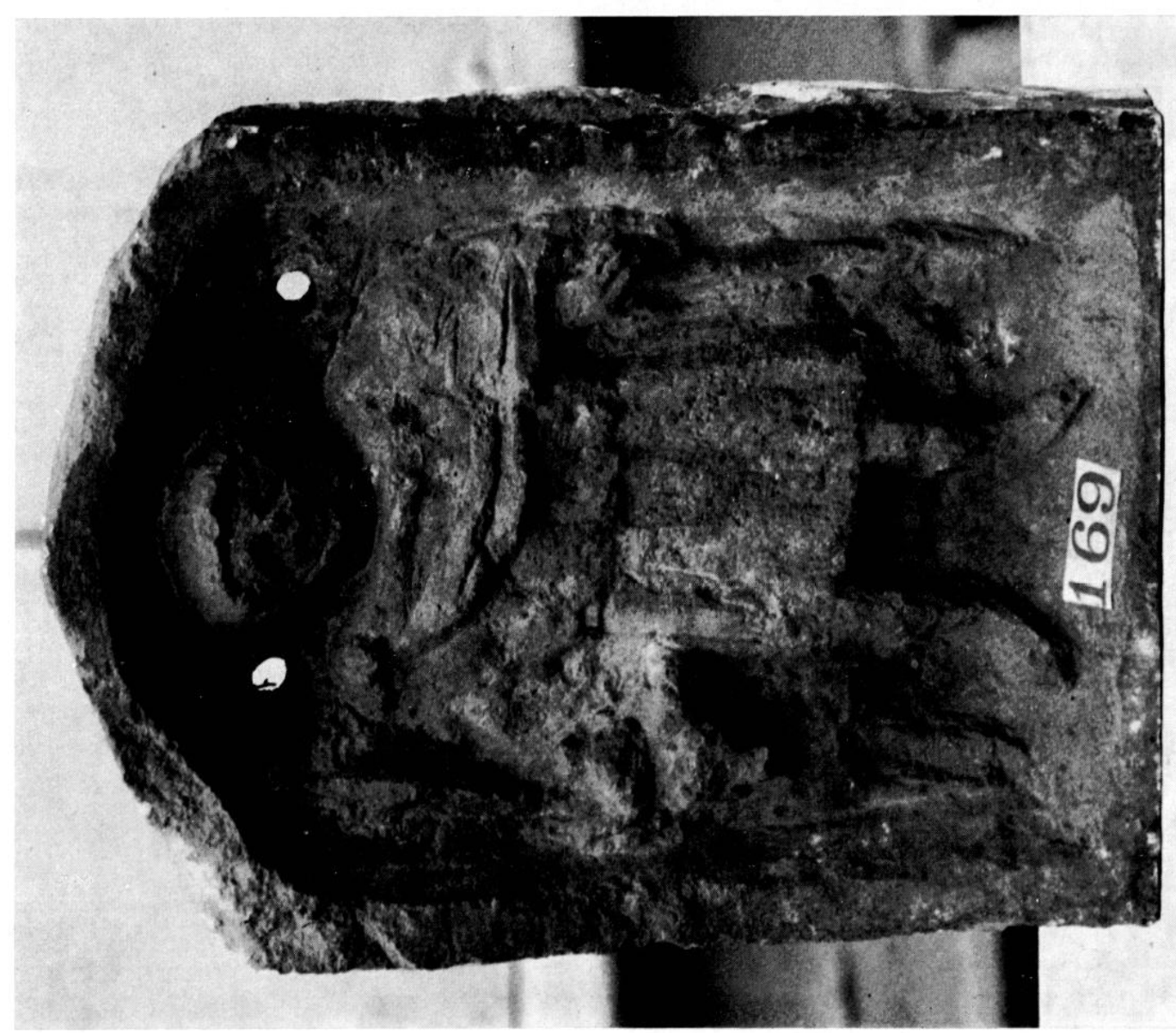

ט 
d'un protedeur lopique ou personnel assorio à quelque Maler ou Tulelle? Pourvu d'altributs banals el identiques à ceux de la déesse. peul-il. au contraire, ètre tenu pour l'interprétation d'un dieu indigene ayant une audience asse\% large. celui dont Mercure est un equivalent? Il a. du Mercure Iraditionnel ${ }^{97}$, la nurlité. l'apparence vigoureuse, el les at tributs qu'il porte symbolisent la prospérité. Il n'y a donc, apparemment, aucune impossibilité formelle à une identification fondée sur des ressemblances. Yous demeurons rependant dans le domaine de l'hypolhese. puisque tous ces "genii" sont malencontreusement prives de leur tite el qu'on ne peut connaitre ni l'aspect de leur visage. ni leur coiffure ${ }^{98}$. Ie plus. il importerait de trouver le moyen de préciser comment on imagrinail le dieu celtique associé à Rosmerta ${ }^{99}$ et l'importance respective qu'ont donc eue, pour favoriser l'interprétation romaine. les similitudes physiques et l'analogie des pouvoirs. Enfin. il faut tenir comple de l'épigraphie locale, qui peul loujours révéler l'existence d'un culle à un Cienius topique ou privét ${ }^{100}$.

Je n’ai envisagé. jusqu'à présent. que l'éventualité selon laquelle nous aurions affaire à deux personnalités divines distinctes. l'une indigene. l'autre romaine, la seconde étant la lransposition d'un dieu celtique inconnu. différent du premier. Le probleme se pose aussi de savoir si la séparation opérée ainsi n'est pas un leurre et si l'on n'a pas. simplement, concurrence entre des formes iconographiques différentes d'une mème réalité. Lous ne disposons d'aucun matériel épigraphique; force est donc de procéder par analogie et de voir comment a pu se manifester plastiquement, dans des cas sans équivoque. l'adaptalion. l'une à l'autre, d'une divinité celtique el d'un dieu romain. Il existe un exemple asse\% net : relui de silvain et du dieu au maillet dans le Vidi de la liance. On constate, en l'occurrence, une contamination évidente des lypes figurés. le dieu au maillet peul ilre nu, comme silvain, ou, comme lui, couronné de feuilles, ou encore. sans changer de costume. se voir doté des attributs de celui-cio1. Observe-t-on une assimilation aussi manifeste entre les "genii" et le dieu au maillet. ou entre ce dernier et Nercure? Le probleme se pose un peu dilléremment selon la divinilé envisagée et les attributs qu'elle porte. Ainsi. la patire el la corne d'abondance sont trés peu caractéristiques: ce sont, en quelque sorte, des attributs génériques et on en fait, de laçon assez large, la propriélé des protecteurs du foyer el surtout de la prospérité. Il n'y a pas lieu, dans ces conditions, de s'élonner de les voir entre les mains de plusieurs divinilés ${ }^{102}$. Aussi me semble-t-il de peu de signification de les trouver. l'une ou l'autre, en la possession du dieu barbu. Il est, intéressant, bien sûr, de pressentir l'existence possible de lamilles dans l'iconographie de ce dieu : patire el bourse ou maillet (fig. 5) ${ }^{103}$. corne d'abondance el olla (ou gobelel 104. Mais jamais, du fail peut-itre de la détérioralion des monuments, on ne trouve le dieu barbu aver la patire el la corne d'abondance (ce qui, comple tenu de la similitude des paridres, permettrail des présomptions plus solides]. pas plus qu'on ne peul saroir si les "genii " el aient ou non imberbesos.

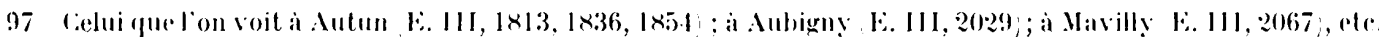
$9 \times$ cif. supra, n. $\times 7$, a propos du dieu de lihangi.

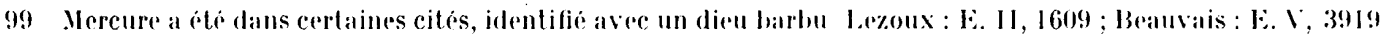
etc. On peut expliquer diversement cette différence : par une contamination plus ou moins poussé entre denx 1 ypes divins en rapport, peut-être, avec une chronologie plus ou moins tardive; par la superposition de llercure a des types locaux distincts d'un même dieu, ou encore à des divinites gauloises differentes.

100 Je probleme demeure, en principe, a peu pres insoluble à moins de termes de comparaison suflisants el pourvus de dédicaces, dans le cas de monuments que leur usage domestique dispensait de porter une inscription.

101 Dien au maillet couromé de feuilles bronze, no 4031 , du musé de Saint-Remy-rle-Provence p portant une

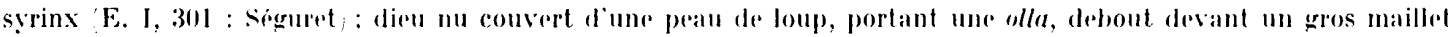

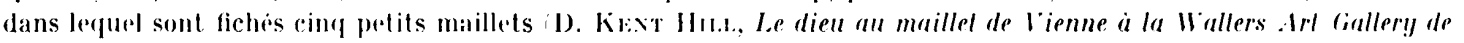
Ballimore, dans Gallia, . 1 1. 195.3, p. 205-22:2.

102 Cif. supra, II. 41 .

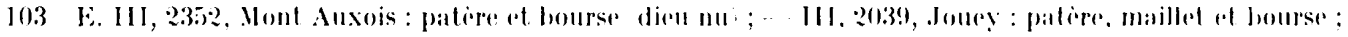

IX, 7121, Mont Auxois : patire on ? maillet ou bourse ?..

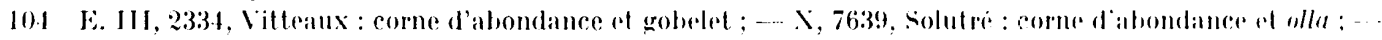
III, 18.37, Autun : cormo dibondance et ?

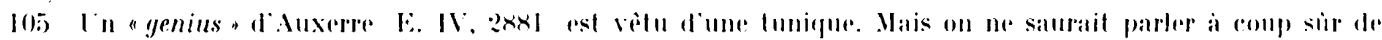

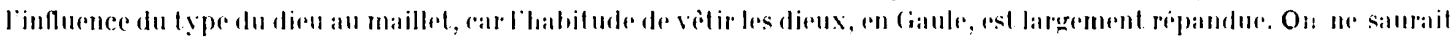


Pour Mercure, la question est aussi peu rlaire, bien que les attributs du dieu romain soient. mieux caraclérisés. Jamais on ne rencontre sur ces reliefs un dieu barbu el vètu qui, comme à leyoux, porte incontestablement la bourse et le caducée $\mathrm{e}^{\mathbf{0 6}}$. Il arrive cependant que le dieu barbu ait une bourse. Ies deux seuls exemples à peu près assurés ont été trouvées à Jouey et au .Iont Auxois ${ }^{\mathbf{1 0 7}}$. On peut se demander, d'abord, dans quelle mesure il ne s'agit pas d'une confusion d'attributs au moins autant que d'une contamination délibérée entre deux types divins : il est surprenant, en eflet, que la bourse n'ait pas la forme allongée de celle qui pend, le plus souvent, au bout du bras de Vercure, mais soit ronde et gonflée, avec un petit bourrelet au-dessus de l'étranglement, c'est-à-dire très semblable au vase sphérique ou ovoïde avec un court rebord que l'on voit, d'ordinaire, dans la main du dieu au maillet ou à ses pieds. De plus, chaque fois, on se trouve en présence d'un mélange complexe d'attributs mettant en cause au moins trois dieux, dont l'identité n'est d'ailleurs pas toujours assuré $\mathrm{e}^{108}$. Aussi, mème si l'on ne conteste pas l'existence de reliefs du dieu barbu avec une bourse, semble-t-il possible de se demander, en chaque occasion, s'il ne s'agil pas d'un type local original, plutòt que de la simple syncrèse de deux divinités, et si à cette iconographie particulière ne correspondait pas une dénomination éqalement particulière de la divinité, et, par conséquent, de sa paridre.

Ainsi il importe de dégager, comme l'ont fait E. Espérandieu et J. Toutain, les (aractéristiques générales d'un culte qui s'adressait à un couple de "bons génies "109, de mettre en évidence, également, le caractère régional de la représentation, sur la plupart des stèles, de divinités assises. Ies analyses de P. Lambrechts ${ }^{110}$ ont montré de façon convaincante comment on pouvait, d'après l'iconographie en particulier, saisir la complexité ot la variété des pouvoirs de rertains dieux celtiques. Toutefois, on parvient presque inévitablement, par cette recomposition l'une personnalité aux aspects divers, à travers une iconographic multiforme, a définir une fonclion divine. Mais on n'est jamais sûr, et P. Lambrechts le dit ${ }^{111}$, de connaître chaque fois l'identité locale du dieu en question. On n'est jamais certain, non plus - et cela, P. Lambrechts l'exprime moins clairement -de définir un dieu qui (à (aause de la diversité même qu'on lui prète) ait jamais existé pour personne. Et le fait de le baptiser, mème avec beaucoup de réserves et une certaine circons-

même, a la limile, être ohligre de supposer une influence gauloise, car il arrive au genius romain de porter la tunigue. Je vêtement, a lui seul, ne pent donc fournir la preuve deune contamination cf. fig. $4 \mathrm{~b}$.

106 (.f. supra, n. 99.

107 Cif. supra, n. 103. Diutres exemples sont trop douteux pour être retenus : E. III, 22.49, 2:312, 23.16..

108 Ine stele de Beaune (E. JII, 2082) pose des problemes analogues. J'y distingue un homme barbu, delout, vêtu d'une courte tunique et d'un manteau. Appuyé de la main gauche sur un bàton, il tient dans la droite un ohjet sphérique au-dessus de la tête d'un animal d'assez petite taille, un quadrupéde représenté de face. Derrière l'épaule droite: une lance ?). Je ne parviens pas à discerner le bouc,? el le chien qui devraient, d'apres la description du catalogue, se trouver à droite du personnage, pas plus que je ne saurais aflirmer que lobjel splierique est une bourse plutot qu'un vase. On voit le dieu au maillet, avec son chien, dans la même attitude, semblablement vêtu sur plusieurs reliefs par exemple : E. I, 435, 436, 437, à Nimes). Le traval de la pierre de Beaune me paraît trop fruste pour qu'on puisse reconnaitre avec une absolue certitude tel animal ou tel objet, plutót qu un autre. Aussi me semble-t-il hasardeux dy chercher la prenve de la contamination du type de Mercure et de cehui de Sucellus, au-dela de la ressemblance, qu'on ne peut contester, entre l'allilude de Mercure tenant une bourse au-dessus de la tête du bouc et celle de sucellus tenant une olla au-dessus de la tête du chien icf. fig. 6 a ot b.

109 J. Tor Tax, Les diviniles domesliques..., p. 420.

110 Op.cit., "Le dieu celtique au maillet" p. 100-116/, "Le couple divin en pa!s idluen" p. 117-120", Recherches sur le culte de Mercure el dr Nars en Gaule " p. 1:21-156;.

111 Ihid., p. 120. 
pection, par exemple Dispater ${ }^{112}$, peut être, à la limite, dangereux, d'autant plus qu'on joue là, selon des modalités variables, tantôt sur l'analogie présuméc des pouvoirs, tantôt sur la ressemblance typologique. C'est pourquoi il m'a paru intéressant de préciser, autant que cela m'était possible, les limites dans lesquelles l'iconographie permettait, dans la région de la cité des Éduens, de supposer dans certains couples figurés l'interprétation romaine d'une divinité masculine indigène. Il devenait plus aisé, ensuite, de savoir si la divinité féminine de ces groupes, toujours identique, par son type, à la déesse d'Escolives, avait ou non des chances d'avoir été connue sous le nom de Rosmerla. L'examen que j'ai tenté me paraît avoir montré que la diversité des types indigènes, à l'intérieur d'un même groupe : celui des dieux barbus, n'interdisait nullement de supposer l'emploi de noms locaux ou, au moins, d'épithètes locales, correspondant, dans un culte populaire, à une nuance particulière de la personnalité, ou de la fonction divine envisagée. Ce qui risquait, normalement, d'impliquer une certaine variété dans les dénominations de la déesse parèdre. Il est apparu, d'autre part, que l'interpénétration des types romains et gaulois n'était pas assez évidente, à cause, en particulier de la détérioration des monuments, pour que l'on puisse avoir la certitude de l'existence d'un seul dieu indigène sous des aspects divers. Faut-il chercher une explication dans le fait que ces types figurés se seraient succédé au licu d'être concurrents? Les difficultés que l'on rencontre pour dater ces petits reliefs, souvent très rustiques et d'origine artisanale, interdisent actuellement de soutenir comme de rejeter cette hypothèse. On peut simplement s'étonner que, dans le cas d'une succession dans le temps, on ne puisse pas déceler de types de transition. Finalement, dans l'ordre des probabilités, l'identification de Mercure et des "genii" à un même dieu indigène ne serait pas à exclure, compte tenu des monuments que nous possédons actuellement et de l'état de ceux-ci ; en revanche, l'utilisation d'une iconographie tantôt romaine, tantôt gauloise, pour la représentation d'une seule divinité, parait beaucoup plus douteuse.

Aussi l'extension du culte de Rosmerla, dans la mesure où celle-ci n'est connue épigraphiquement que comme parèdre de Mercure, est-elle à envisager avec prudence. Nous avions déjà vu comment l'identification de cette déesse avec certaines Malres demeurait hypothétique. Nous constatons, maintenant, toutes les difficultés que présente son association avec des dieux analogues à Mercure par leur fonction et, sans doute, parfois, les modalités de leur culte, mais différents par leurs attributs. On connaît, à Escolives, ce qui paraît être un dieu Smertu [-- - $]^{113}$. La présence, dans ce nom incomplet, du radical *smer- rend

112 Ibid., "Peu nous importe d'ailleurs le nom qu'il sied de donner à ces divinités. Ce qui nous intéresse, c'est de savoir que nous avons affaire à une expression plastique, très localisée - et peut-être inspirée de modèles classiques, comme le prouveraient les monuments correspondants de Jupiter et de Junon à Mayence - du grand dieu celtique Iispaler. "

113 Photographie publiée, avec une petite note signée A. C., dans Pays de Bourgogne, no 56, 1967, p. 487. L'autel, utilisé en remploi, comme me l'a précisé .I. R. Kapps, a été retaillé de telle faron qu'on peut imaginer qu'il manque à la fin des lignes, supposées complètes, deux ou trois lettres. Le nom SMERTV - - - doit-il être complété en Smertul( ()$_{0}$ ou Smertuco, d'après les anthroponymes attestés : Smerlullus et Smerluca (et Smerluccus?) cf. P.M. Duval, Le dieu Smertrios el ses alalars gallo-romains, dans Eludes celliques, VI, no 12, 1952, p. 219-238) ? (''est ce que je ne saurais dire. 
tentante une éventuelle association avec $R_{0}\left(-\right.$ smerta $^{114}$. Est-ce lui qu'on a représenté sous l'apparence de Mercure? Avait-il un ou plusieurs autres visages ${ }^{115}$ ? Seul l'heureux hasard de nouvelles découvertes sur 'e site pourrait nous apporter quelques certiturles.

Colette Bímont.

114 Si l'on songe aux jeux auxquels prête le nom des dieux de certains couples (l'isucius / Visucia, Bormanus ) Bormana, cités par P. Lambrecirs, op. cil., p. 185̃).

115 Les épithetes les plus voisines appliquées aux dieux gaulois sont smeririos, qui qualifie, au moins une fois, Mars, à Liesenich, chez les Trévires (CIL, XIII, 11975), et Adsmcrios, appliqué à Mercure à Poitiers (CII, XIII, 1125). (.f. P.-.I. Drvar, op. cit., p. 224-225. 